\title{
Fading AGN Candidates: AGN Histories and Outflow Signatures*
}

\author{
William C. Keel ${ }^{1}$, Chris J. Lintott ${ }^{2}$, W. Peter Maksym ${ }^{1,3}$, Vardha N. Bennert ${ }^{4}$, S. Drew Chojnowski ${ }^{5}$, Alexei Moiseev ${ }^{6}$, \\ Aleksandrina Smirnova ${ }^{6}$, Kevin Schawinski ${ }^{7}$, Lia F. Sartori ${ }^{7}$, C. Megan Urry ${ }^{8}$, Anna Pancoast ${ }^{3,10}$, Mischa Schirmer ${ }^{9}$, Bryan Scott ${ }^{4}$, \\ Charles Showley ${ }^{4}$, and Kelsi Flatland ${ }^{4}$ \\ ${ }^{1}$ Department of Physics and Astronomy, University of Alabama, Box 870324, Tuscaloosa, AL 35487, USA; wkeel@ua.edu \\ ${ }^{2}$ Astrophysics, Oxford University; and Adler Planetarium, 1300 S. Lakeshore Drive, Chicago, IL 60605, USA \\ ${ }^{3}$ Center for Astrophysics, 60 Garden St., Cambridge, MA 02138, USA \\ ${ }^{4}$ Physics Department, California Polytechnic State University, San Luis Obispo, CA 93407, USA \\ 5 Department of Astronomy, New Mexico State University, P.O. Box 30001, MSC 4500, Las Cruces, NM 88003-8001, USA \\ ${ }^{6}$ Special Astrophysical Observatory, Russian Academy of Sciences, Nizhny Arkhyz, 369167, Russia \\ ${ }_{8}^{7}$ Institute for Astronomy, ETH Zürich, Wolfgang-Pauli-Straße 27, CH-8093 Zurich, Switzerland \\ ${ }^{8}$ Department of Physics, Yale University, P.O. Box 208120, New Haven, CT 06520-8120, USA \\ ${ }^{9}$ Gemini Observatory, La Serena, Chile \\ Received 2016 August 31; revised 2016 December 2; accepted 2016 December 18; published 2017 February 1
}

\begin{abstract}
We consider the energy budgets and radiative history of eight fading active galactic nuclei (AGNs), identified from an energy shortfall between the requirements to ionize very extended (radius $>10 \mathrm{kpc}$ ) ionized clouds and the luminosity of the nucleus as we view it directly. All show evidence of significant fading on timescales of $\approx 50,000 \mathrm{yr}$. We explore the use of minimum ionizing luminosity $Q_{\text {ion }}$ derived from photoionization balance in the brightest pixels in $\mathrm{H} \alpha$ at each projected radius. Tests using presumably constant Palomar-Green QSOs, and one of our targets with detailed photoionization modeling, suggest that we can derive useful histories of individual AGNs, with the caveat that the minimum ionizing luminosity is always an underestimate and subject to uncertainties about fine structure in the ionized material. These consistency tests suggest that the degree of underestimation from the upper envelope of reconstructed $Q_{\text {ion }}$ values is roughly constant for a given object and therefore does not prevent such derivation. The AGNs in our sample show a range of behaviors, with rapid drops and standstills; the common feature is a rapid drop in the last $\approx 2 \times 10^{4} \mathrm{yr}$ before the direct view of the nucleus. The $e$-folding timescales for ionizing luminosity are mostly in the thousands of years, with a few episodes as short as 400 yr. In the limit of largely obscured AGNs, we find additional evidence for fading from the shortfall between even the lower limits from recombination balance and the maximum luminosities derived from far-infrared fluxes. We compare these long-term light curves, and the occurrence of these fading objects among all optically identified AGNs, to simulations of AGN accretion; the strongest variations over these timespans are seen in models with strong and local (parsec-scale) feedback. We present Gemini integral-field optical spectroscopy, which shows a very limited role for outflows in these ionized structures. While rings and loops of emission, morphologically suggestive of outflow, are common, their kinematic structure shows some to be in regular rotation. UGC 7342 exhibits local signatures of outflows $<300 \mathrm{~km} \mathrm{~s}^{-1}$, largely associated with very diffuse emission, and possibly entraining gas in one of the clouds seen in Hubble Space Telescope images. Only in the Teacup AGN do we see outflow signatures of the order of $1000 \mathrm{~km} \mathrm{~s}^{-1}$. In contrast to the extended emission regions around many radio-loud AGNs, the clouds around these fading AGNs consist largely of tidal debris being externally illuminated but not displaced by AGN outflows.
\end{abstract}

Key words: galaxies: active - galaxies: individual (NGC 5792, UGC 7342, Mkn 1498) - galaxies: interactions galaxies: Seyfert

\section{Introduction}

It has long been known that some active galactic nuclei (AGNs) are accompanied by extended emission-line regions (EELRs), zones of ionized gas spanning galaxy scales or even larger. Such regions can trace the geometry of ionizing radiation escaping the AGN and host galaxy, and at least implicitly give hints to the luminosity history of the AGN. EELRs occur around Seyfert nuclei, QSOs, and radio galaxies.

A very luminous and extensive EELR was found in the course of the Galaxy Zoo project (Lintott et al. 2008) near the

\footnotetext{
* Based on observations with the NASA/ESA Hubble Space Telescope obtained at the Space Telescope Science Institute, which is operated by the Association of Universities for Research in Astronomy, Inc., under NASA contract No. NAS5-26555.

${ }^{10}$ Einstein Fellow.
}

spiral galaxy IC 2497. Known after its discoverer as Hanny's Voorwerp, this object shows high-ionization emission lines with ratios essentially identical to the narrow-line region of an AGN, spanning a projected range from 15 to $35 \mathrm{kpc}$ from the galaxy nucleus, which fails by at least two orders of magnitude in bolometric output to match the ionization requirements of the cloud (Lintott et al. 2009; Keel et al. 2012b). The cloud's electron temperature, narrow line widths, and quiescent velocity field indicate photoionization rather than shocks as the energy source. H I mapping by Józsa et al. (2009) shows this to be the ionized part of a $300 \mathrm{kpc}$ trail of otherwise neutral gas, suggesting a strong galaxy interaction gigayears ago. The energy mismatch between the nucleus of IC 2497 and the ionization requirements in Hanny's Voorwerp indicates that the nucleus faded from a luminosity associated with QSOs to a modest Seyfert or LINER level within $10^{5} \mathrm{yr}$. 
Starting with the unusual morphology and Sloan Digital Sky Survey (SDSS) gri colors of Hanny's Voorwerp, a targeted search by Galaxy Zoo volunteers found 19 additional EELRs, including hitherto unknown examples, of which eight showed energy shortfalls similar to that in IC 2497 (Keel et al. 2012a). This sample was selected in a homogeneous way, requiring detection of emission features showing AGN-like line ratios more than $10 \mathrm{kpc}$ in projection from the nucleus. We have pursued a detailed study of this subset of eight (plus IC 2497/ Hanny's Voorwerp) to learn more about AGN variations on the otherwise inaccessible range of timescales of $10^{4}-10^{5} \mathrm{yr}$. Analogous cases of extended ionized gas near energetically inadequate AGNs have been reported at higher redshift and luminosity by Schirmer et al. (2013, 2016), their "Green Bean" systems, and at low luminosity in the local universe in the merger remnant NGC 7252 (Schweizer et al. 2013) and as one interpretation of an off-nuclear ionized region in the spiral galaxy NGC 3621 (Menezes et al. 2016).

Interest in the history of accretion onto nuclear black holes has also been renewed by evidence for an eventful history of outbursts within $10^{6} \mathrm{yr}$ by the currently quiescent black hole at the Galactic Center, from X-ray echoes (Muno et al. 2007), the ionization structure of the Magellanic Stream (Bland-Hawthorn et al. 2013), and possibly the "Fermi bubbles" (Zubovas et al. 2011; Guo \& Mathews 2012).

The variations in AGN luminosity we infer from these objects, spanning scales up to $10^{5} \mathrm{yr}$, connect implicitly with the variations on timescales we can observe directly. "Changing-look" AGNs can have broad-line emission essentially vanish over $\approx 10 \mathrm{yr}$ in the emitted frame (MacLeod et al. 2015; Ruan et al. 2015; Runco et al. 2016; Runnoe et al. 2016), while the narrow-line region is often so large that light-travel-time smearing leaves its emission nearly constant; similar interpretations were discussed in the context of spectral variations in Seyfert nuclei at least as early as Penston \& Perez (1984). The temporal spectrum of variations in AGN output clearly spans many orders of magnitude, and affects our understanding not only of their physical structure but also of the demographics of accretion in the galaxy population. Seeing dim episodes so long that they appear in surrounding ionized gas when smeared by the recombination timescales of thousands of years, as well as geometric projection factors, means either that the AGNs stay in a very low state for these long times or that excursions to a high state fill only a small fraction of the time. Understanding this history is key to understanding the broad idea of duty cycles in AGN accretion; X-ray surveys in particular suggest a duty cycle connected to the Eddington limit and evolving with cosmic time (e.g., Shankar et al. 2009), but not how many episodes of what durations are involved.

In a similar sense, radio observations suggest very episodic production of jets, a major form of kinetic energy output from accretion. The identification of radio jets and a circumnuclear outflow in IC 2497 (Józsa et al. 2009; Rampadarath et al. 2010; Keel et al. 2012b) led us to speculate that some of the extreme variability needed to explain the ionization of Hanny's Voorwerp might come not solely from a drop in the accretiondriven luminosity of the AGN in IC 2497, but from some of the "missing" luminosity switching to a kinetic mode. This idea is supported by a "bubble" of hot gas around the fading AGN of IC 2497 seen with Chandra (Sartori et al. 2016).

All these factors motivated more detailed study of our fading-AGN candidates with a variety of techniques. Paper I
(Keel et al. 2015) presented results of Hubble Space Telescope (HST) imaging concerning the host galaxies and the origin of the ionized gas. The hosts are bulge-dominated, and every one shows signatures of ongoing or past galaxy interactions, 1.5-3 Gyr ago for systems with favorable geometry to estimate this timescale. The extended gas has modestly subsolar metallicity, and is kinematically rotation-dominated; outflows have only a very localized role, in contrast to most QSO EELRs (Stockton et al. 2006). These are externally illuminated tidal debris; merger remnants are particularly good environments for $\mathrm{H}$ I to occur far from the nucleus and out of any host disk plane, and act as a screen to show the escaping ionizing radiation. This appears to be such a strong selection factor that we do not know whether high-amplitude variability on scales of $10^{4} \mathrm{yr}$ is confined to merger aftermaths or not.

In this paper, we describe additional data, including Gemini integral-field spectroscopy, and present implications of the entire data set for the properties and history of these AGNs. We use the peak surface brightness in recombination lines at various projected radii to reconstruct the luminosity history of the AGNs, compare structures seen in the emission-line images and line ratios to address the role of ionization cones, and examine the occurrence of outflows near the nuclei to trace the role and extent of feedback near the epochs of radiative dimming.

In evaluating sizes and luminosities, we compute distances using a Hubble constant of $73 \mathrm{~km} \mathrm{~s}^{-1} \mathrm{Mpc}^{-1}$.

\section{Observations}

\subsection{Galaxy Sample}

As given by Keel et al. (2015), the sample of AGNs we consider includes the eight systems from the Galaxy Zoo survey of giant ionized clouds (Keel et al. 2012a) where a simple estimate of energy budget including the ionization requirements of the most distant emission-line region, compared to the observed bolometric luminosity of the nuclei, showed a shortfall greater than about a factor 5. These include NGC 5252 with its well-documented set of ionization cones (Prieto \& Freudling 1996; Morse et al. 1998), and the large-scale double radio source hosts NGC 5972 (Véron-Cetty \& Véron 2001) and Mkn 1498 (Röttgering et al. 1996). Additional objects, some showing larger and more luminous EELRs, were newly found in this survey. This work deals with the $\approx 40 \%$ of the Galaxy Zoo EELR objects showing evidence for AGN fading; the remainder have energy budgets indicating roughly constant luminosity in some cases with substantial obscuration along our line of sight. Overall properties of these galaxies are listed in Table 1, including IC 2497 (host galaxy of Hanny's Voorwerp) for reference.

\subsection{HST Imaging}

Central to our analysis is a set of emission-line images of our fading-AGN candidates obtained with the HST and narrowband filters in the Wide Field Camera 3 (WFC3) or tunable ramp filters on the Advanced Camera for Surveys (ACS), using archival WFPC2 data for NGC 5252. These data and our processing are described in detail by Keel et al. (2015). For brevity, we truncate the SDSS designations of two systems without more common catalog names, SDSS J151004.01 +074037.1 and SDSS J220141.64+115124.3. 
Table 1

Galaxy Properties

\begin{tabular}{llccc}
\hline \hline Galaxy & SDSS Name & Type & $z$ & Scale $\left(\mathrm{kpc} \operatorname{arcsec}^{-1}\right)$ \\
\hline IC 2497 & SDSS J094104.11+344358.4 & 2 & 0.0502 & 0.96 \\
Mkn 1498 & SDSS J162804.06+514631.4 & 1.9 & 0.0547 & 0.0228 \\
NGC 5252 & SDSS J133815.86+043233.3 & 1.5 & 0.0297 & 0.11 \\
NGC 5972 & SDSS J153854.16+170134.2 & 2 & 0.0458 & 0.63 \\
SDSS 1510+07 & SDSS J151004.01+074037.1 & 2 & 0.0296 & 0.89 \\
SDSS 2201+11 & SDSS J220141.64+115124.3 & 2 & 0.0852 & 0.59 \\
Teacup AGN & SDSS J143029.88+133912.0 & 2 & 0.0477 & 0.98 \\
UGC 7342 & SDSS J121819.30+291513.0 & 2 & 0.0412 \\
UGC 11185 & SDSS J181611.61+423937.3 & 2 & 0.80 \\
\hline
\end{tabular}

Table 2

WISE Magnitudes and IR Luminosities

\begin{tabular}{lrrrrr}
\hline \hline Galaxy & W1 $(3.4 \mu \mathrm{m})$ & W2 $(4.6 \mu \mathrm{m})$ & W3 $(12 \mu \mathrm{m})$ & W4 $(22 \mu \mathrm{m})$ & Log $L(\mathrm{MIR})$ \\
\hline IC 2497 & $11.46 \pm 0.02$ & $11.15 \pm 0.02$ & $7.31 \pm 0.02$ & $4.54 \pm 0.03$ & 4.28 \\
Mkn 1498 & $10.26 \pm 0.02$ & $9.24 \pm 0.02$ & $6.31 \pm 0.02$ & $3.72 \pm 0.02$ & 45.41 \\
NGC 5252 & $9.19 \pm 0.02$ & $8.38 \pm 0.02$ & $6.35 \pm 0.01$ & $4.47 \pm 0.02$ & 44.77 \\
NGC 5972 & $11.02 \pm 0.02$ & $10.49 \pm 0.02$ & $7.05 \pm 0.02$ & $4.61 \pm 0.02$ & 4.28 \\
SDSS 1510+07 & $12.93 \pm 0.04$ & $12.81 \pm 0.03$ & $11.46 \pm 0.12$ & $>9.00$ & 44.23 \\
SDSS 2201+11 & $11.10 \pm 0.02$ & $11.08 \pm 0.02$ & $8.60 \pm 0.02$ & $6.77 \pm 0.07$ & 43.15 \\
Teacup AGN & $11.67 \pm 0.02$ & $10.50 \pm 0.02$ & $7.14 \pm 0.01$ & $4.16 \pm 0.02$ & 43.57 \\
UGC 7342 & $12.70 \pm 0.02$ & $12.61 \pm 0.03$ & $9.90 \pm 0.05$ & $7.17 \pm 0.10$ & 45.42 \\
UGC 11185 & $11.22 \pm 0.02$ & $10.61 \pm 0.02$ & $7.85 \pm 0.02$ & $4.88 \pm 0.02$ & 43.70 \\
\hline
\end{tabular}

Note. WISE data are magnitudes in filters W1-W4. Luminosities are computed for the ranges 3.4-42 $\mu \mathrm{m}$ for MIR and $42-122 \mu \mathrm{m}$ for FIR, expressed as the decimal $\log$ of the values in erg $\mathrm{s}^{-1}$.

\subsection{WISE Data}

Our estimates of the bolometric luminosity of the AGNs, and hence the effect of obscuration on the ionizing radiation, can be updated from Keel et al. (2012a) using the more sensitive and higher-resolution survey data from the Wide-field Infrared Survey Explorer (WISE, Wright et al. 2010) in place of IRAS or Akari for the mid-infrared region including $22 \mu \mathrm{m}$. WISE data show that much of the mid- and far-IR flux attributed initially to UGC 7342 at $z=0.047$ comes instead from a background starburst system at $z=0.069$ (as identified in Paper I). The AGN luminosity we derive for UGC 7342 is correspondingly lower, and the energy deficit for ionizing its gas filaments becomes even greater.

The WISE catalog magnitudes are listed in Table 2. For further use, we converted them into fluxes using the zero points from Cutri et al. (2014) for "compromise" spectral slopes.

The WISE data are especially important since much of the reradiation from circumnuclear dust occurs in the mid-IR, which was poorly covered by previous surveys. The infrared output of these galaxies, and thus the potential fraction of obscured and reradiated AGN radiation, can be quantified in several ways. We have derived total fluxes and luminosities by integrating power-law spectra connecting the data points and extrapolating to the boundaries of common bands, and by fitting the data to various template spectral energy distributions derived for AGNs. Table 2 includes mid-IR luminosities. These are given as the log of the value in erg s${ }^{-1}$ calculated by powerlaw interpolation between the WISE bands, with a redward extrapolation to span from 3.4-42 $\mu \mathrm{m}$ for comparison with the IRAS-based FIR luminosity as listed in Keel et al. (2012a), which includes the range $42-122 \mu \mathrm{m}$ (Fullmer \& Lonsdale 1989). SDSS $1510+07$ is undetected in the longest WISE band; the listed luminosity assumes the true flux is at this limit, while setting the flux to zero gives a luminosity 0.14 smaller in the log. For comparison, we also list the $L(\mathrm{FIR})$ value (likewise, decimal $\log$ of the value in erg $\mathrm{s}^{-1}$ ) from Keel et al. (2012a). Many of these objects have mid-IR luminosities much greater than the FIR values, high enough in Mkn 1498 and the Teacup system to leave open the possibility of an obscured rather than fading AGN in the absence of other evidence (such as the recombination histories we calculate below). In the others, even including the MIR range often most sensitive to AGN heating of surrounding grains, an order-ofmagnitude energy mismatch is seen between the AGN itself and the requirement to ionize distant clouds. For UGC 7342 and SDSS $2201+11$, the shortfall exceeds a factor 100 . These luminosity estimates are considered in comparison to ionization-based values in Section 4.3.

\subsection{Spectroscopic Mapping}

We investigate the kinematics and ionization structure near the nuclei of several of these galaxies using integral-field optical spectroscopy from the Gemini Multiple-Object Spectrometer (GMOS) system (Davies et al. 1997) at the $8 \mathrm{~m}$ Gillette Gemini-N telescope on Maunakea, under programs GN2013B-Q-25 and GN-2014A-Q-25. The GMOS integral-field unit (IFU) samples the sky in 0 ". 2 apertures covering a region roughly 3 ." $5 \times 5^{\prime \prime}$ in the single-slit mode we used (AllingtonSmith et al. 2002). The B600 grating covered a wavelength range usually encompassing from $\mathrm{He}$ II $\lambda 4686$ to the [S II] $\lambda \lambda 6717,6731$ lines, adjusted for each object's redshift, at $0.468 \AA$ per pixel. Three stepped grating settings, usually at $200 \AA$ intervals, were used to fill gaps between the three GMOS CCDs, with two 20 minute exposures at each step to identify cosmic-ray events. Reduction of these data followed the 
Table 3

Gemini GMOS IFU Spectra

\begin{tabular}{|c|c|c|c|c|}
\hline Location & Offsets (arcsec) & UT dates & FWHM (arcsec) & Observers \\
\hline UGC 11185 pos 1 & $0.2 \mathrm{~W} 3.4 \mathrm{~S}$ & 2013 Aug 13, 27 & 0.48 & M. Hoenig \\
\hline UGC 11185 pos 2 & $2.5 \mathrm{E} 1.2 \mathrm{~S}$ & 2014 Aug 13 & 0.41 & A.-N. Chene \\
\hline UGC 7342 pos 1 & $1.7 \mathrm{~N}$ & 2014 Jun 26 & 0.79 & J. Chavez \\
\hline UGC 7342 pos 2 & $1.7 \mathrm{~S}$ & 2014 Jun 24 & 0.76 & M. Pohlen \\
\hline Mkn 1498 & 0 & 2014 Jul 26 & 0.40 & L. Fuhrman \\
\hline NGC 5972 & $0.3 \mathrm{E}$ & 2014 Jun 26 & 0.47 & J. Chavez \\
\hline Teacup ENE & 4.02 E $2.30 \mathrm{~N}$ & $2014 \mathrm{Jul} 27$ & 0.56 & L. Fuhrman \\
\hline Teacup E & 2.78 E $0.04 \mathrm{~N}$ & 2014 Jul 18 & 0.44 & M. Pohlen \\
\hline Teacup SW & $1.5 \mathrm{~W} 0.63 \mathrm{~S}$ & 2014 Jun 30 & 0.45 & J. Ball \\
\hline
\end{tabular}

procedures described by Davies et al. (2015), including correction for internally scattered light and use of LACOSMIC (van Dokkum 2001) to reject pixels contaminated by cosmic rays. The rms scatter of measured comparison-line wavelengths across the whole array was $0.08 \AA, 5 \mathrm{~km} \mathrm{~s}^{-1}$ for [O III], so the accuracy of this calibration is much finer than the amplitudes of velocity structures we trace. Data from each observation were combined into a data cube evenly sampled on the sky, taking into account atmospheric dispersion. Data cubes from all three grating positions were combined at the end to provide an additional level of rejection for cosmic rays and other sources of bad pixels. Flux calibration used a single observation of $\mathrm{HZ}$ 44; as the observations occurred on dates from 2013 August 13 to 2014 August 27, this flux calibration will be only approximate. We do have external checks on line ratios based on previous long-slit spectroscopy (Keel et al. 2012a, 2015). Both UGC 11185 data runs were truncated by deteriorating conditions; we have only a single grating tilt for each IFU position, with $2 \times 1200 \mathrm{~s}$ exposures, and these are affected significantly by cosmetic issues, so coverage of some spectral lines is incomplete and their flux scales are unreliable.

The delivered image quality ranged from 0 ". 4 to 0 ". 8 FWHM during these observations, as estimated from red-light ( $r$-band) acquisition images obtained immediately before the spectral data. Most of these observations had FWHM $<0$." 5 . As listed in Table 3, we have observations centered on the nuclei of Mkn 1498 and NGC 5972, and sets of two overlapping offset fields for UGC 7342 and 11185; the table includes the requested offsets from the galaxy nucleus to the observed IFU center point. For the Teacup AGN, three offset fields cover virtually all the emission-line regions seen in the HST ACS images (Keel et al. 2015). Similar data for this galaxy taken with the ESO VIMOS system have been shown by Harrison et al. (2015); they describe results only for the [O III] emission lines, and the spatial scale of 0 " 67 per pixel limits their spatial resolution compared to these GMOS data.

For straightforward comparison of emission-line properties over wide ranges in signal-to-noise ratio $(\mathrm{S} / \mathrm{N})$, we fit Gaussian profiles to the lines, constraining $\mathrm{H} \alpha$ and the adjacent [N III] lines to have the same FWHM, with the same constraint on the [S II] doublet. In some regions, each line is double or triple, so we fit blended sets of Gaussians; we used the IRAF task splot interactively on the data cubes sampled in 0 !! 2 spatial increments. Line properties of interest (flux, wavelength, FWHM) were gridded into spatial maps for further analysis. In most cases, two-component fits were constrained to have the same FWHM, for consistency across a wide range in $\mathrm{S} / \mathrm{N}$; for the narrower profiles dominated by the instrumental resolution, this is a good fit. Elsewhere, there may be systematics introduced, but to first order the flux in each component is preserved.

\section{Ionization Geometry}

Some of these cloud systems show plausible ionization cones, briefly mentioned in Paper I. In Figure 1 we show lineratio images of $[\mathrm{O} \mathrm{III}] / \mathrm{H} \alpha$, which often map these structures more clearly than [O III] intensity alone. Among our galaxies, some show cone-like structures in line intensity; we see a few where the line ratio maps ionization in a clearer structure. Most notably, in the Teacup AGN there is a reasonably well-defined triangular region of higher ionization (where the "hole" lies within it). In others, such as UGC 7342 and UGC 11185 (Hainline et al. 2016), the entire emission region could plausibly show a biconical structure without any internal differences in ionization. In these two systems, radial changes in the line ratio are greater than azimuthal variations at fixed projected radius.

Broadly, all these emission complexes show a twofold symmetry, as sketched in Keel et al. (2012a) from early data. When their outer parts resemble ionization cones, we often see much broader regions near the core (often completely encompassing the AGN), whose ionization may have a distinct mechanism.

The emission region in Mkn 1498 is highly elongated but not biconical. Among the remainder, most of the opening angles implied by the HST data are close to our initial estimates, except for the Teacup AGN where we now recognize an inner structure traced by the $[\mathrm{O} \mathrm{III}] / \mathrm{H} \alpha$ ratio for which each half has a full angle of $37^{\circ}$. In SDSS $2201+11$, we derive $25^{\circ}$, although this may be affected by obscuration within the host galaxy on one side of each cloud. The rest are broader, with NGC 5252 at $64^{\circ}$, UGC 7342 at $75^{\circ}$, and NGC 5972 at $96^{\circ}$ to encompass the outer structures. The broadest are seen in UGC $11185\left(116^{\circ}\right)$ and SDSS $1510+07\left(126^{\circ}\right)$. Projection effects in a conical distribution make this angle appear larger than its threedimensional value.

Side-to-side asymmetries in ionization (as in BPT plots, or in reconstructed ionization history) could in principle record front-back differences in light travel time when seen at a sharp angle to the axis of ionization cones or radially elongated clouds. We do not actually see any such differences; our selection for large transverse extent favors cloud systems viewed near the plane of the sky.

In SDSS 2201+11, a dust lane is continuous with the emission-line material into the northern cloud, showing that the northern cloud must be at the front of the system in order to give significant dust attenuation. 


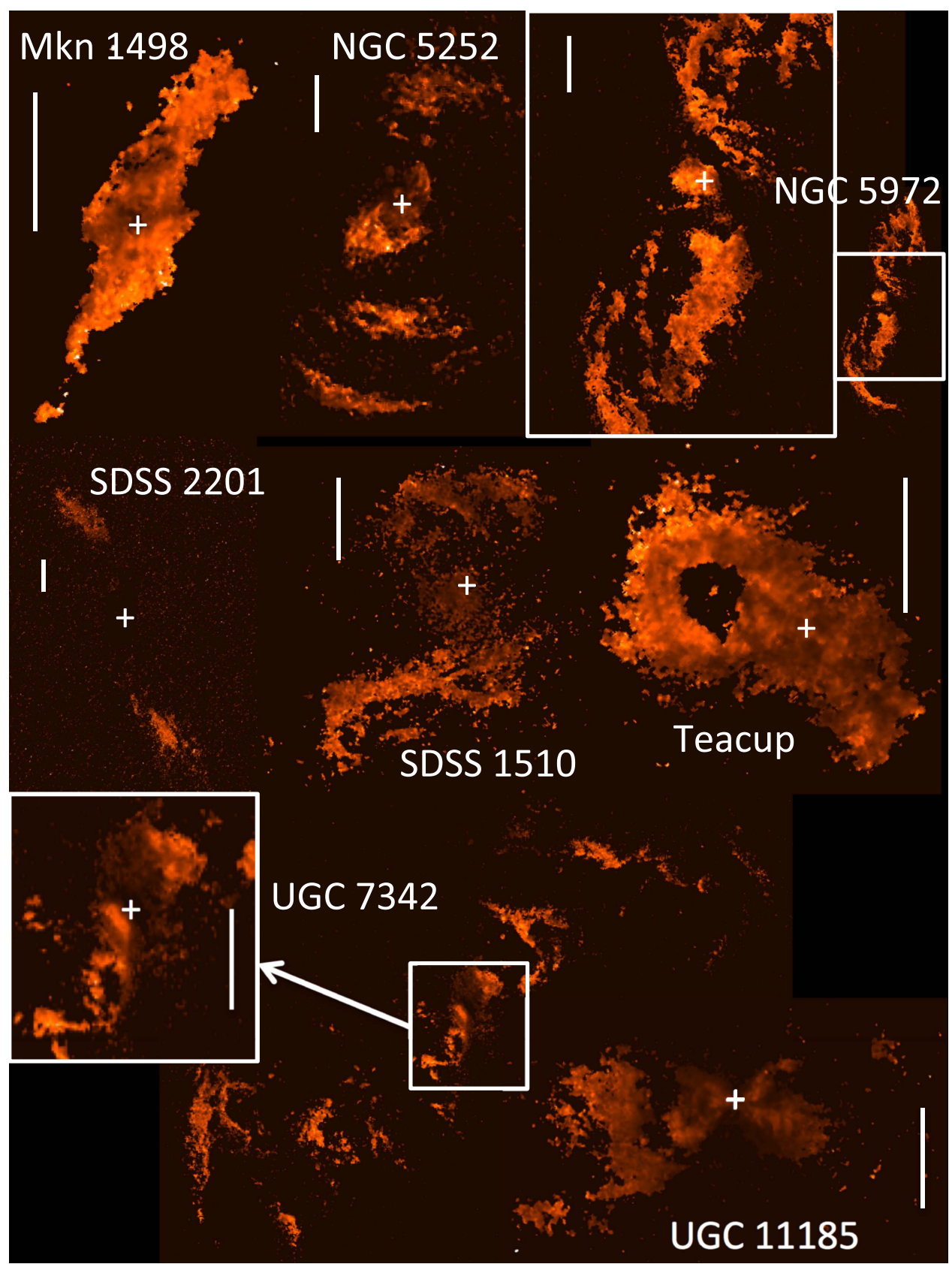

Figure 1. $[\mathrm{O}$ III $] / \mathrm{H} \alpha$ line-ratio images, using a "blackbody" color palette to increase the visual dynamic range and to show features dimmer than easily appear with a pure grayscale. The scale is the same for all, running from zero to a line ratio of 3.6 shown as white. The images were masked at $2 \sigma$ in $\mathrm{H} \alpha$ intensity (the weaker line in most areas), then a $3 \times 3$ pixel median filter was used to suppress noise since the line ratio is more consistent across pixels than the intensities. North is at the top and east to the left; the angular scale varies as shown by $5^{\prime \prime}$ scale bars. For the large cloud systems of NGC 5972 and UGC 7342, insets show where the enlarged nuclear regions are located. The nuclear region of SDSS 2201 is affected by reddening from a dust lane, so no reliable line ratios are available in the inner parts. The location of each AGN is shown by a plus sign.

Near the nuclei, and outside the ionization cones, several of these galaxies show gas with low ionization as seen in $[\mathrm{O}$ III $] / \mathrm{H} \alpha$. One goal of the GMOS IFU spectra was to measure additional line ratios in these regions to probe their ionization mechanism. We will explore this issue elsewhere, in conjunction with recent spectra from the HST Space Telescope Imaging Spectrograph that isolate key regions near selected nuclei.

Overall, the occurrence of ionization cones pointing to the nucleus suggests that the ionized gas has distributions that are mostly radial, rather than, for example, ringlike. This means that projected distance from the nucleus can serve as a useful proxy for physical separation from the AGN in these clouds.

\section{AGN Luminosity History}

\subsection{Method: Recombination Balance}

We explore here the use of recombination-balance arguments to provide lower limits to the ionizing flux reaching various parts of the AGN emission regions. Except in areas where we see the superposition of multiple emission regions along the line of sight, or similarly, elongated filaments seen 


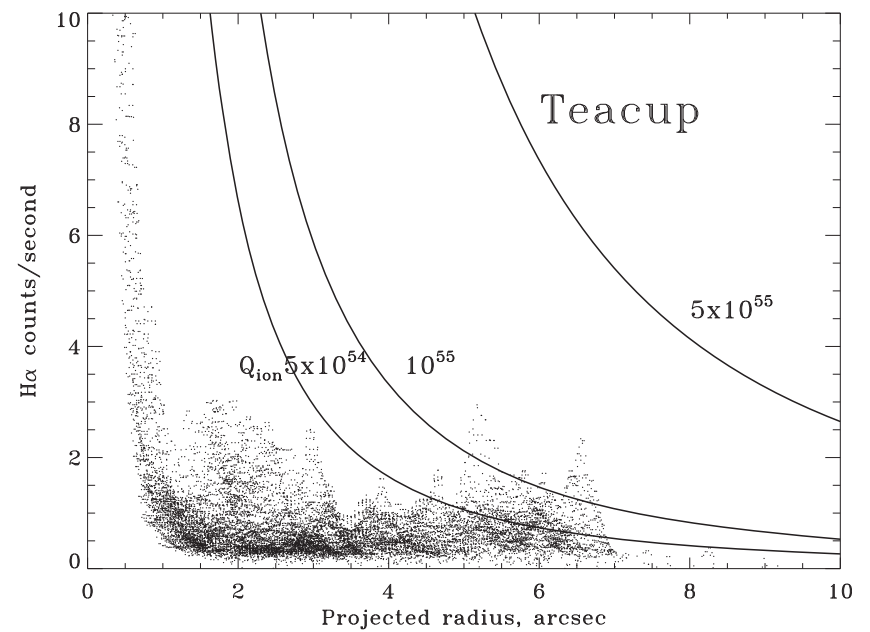

Figure 2. Radial plot of pixel-by-pixel surface brightness (in instrumental units, detected photons per second per pixel) in $\mathrm{H} \alpha$ for the Teacup AGN, with superimposed curves of constant $Q_{\text {ion }}$ in photons s ${ }^{-1}$. Median filtering over $3 \times 3$ pixels was used to suppress residual cosmic-ray and charge-transfer effects, with the side effect of creating correlations between some sets of adjacent points.

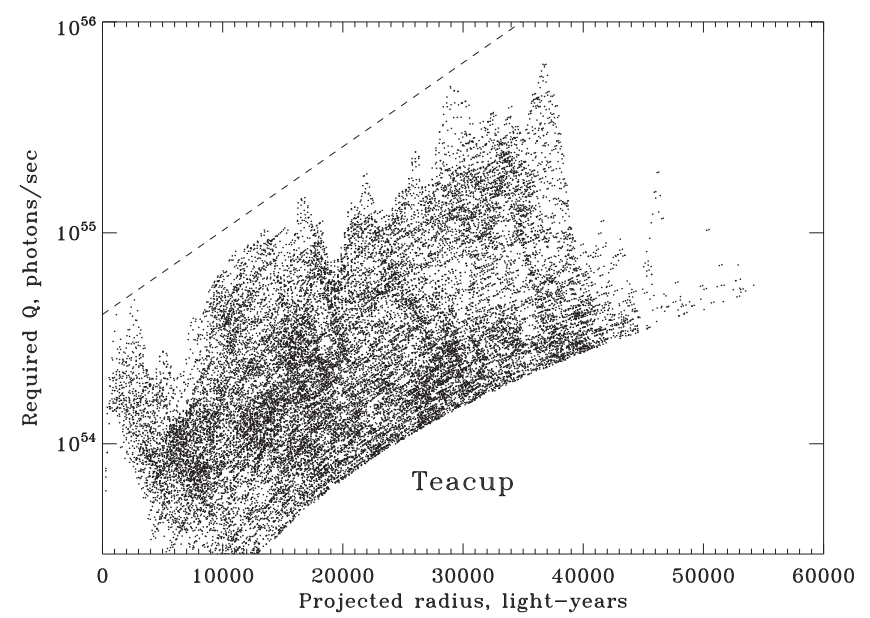

Figure 3. Radial plot of pixel-by-pixel minimum required ionizing luminosity for the Teacup AGN, derived from the data in Figure 2. Projected distance from the AGN is expressed in light-years to make the timescales apparent. The dashed line at the top represents the ionization history inferred independently by Gagne et al. (2014) from detailed modeling of ground-based spectra. The envelope at the bottom represents a $2 \sigma$ cut to suppress the effects of pixel noise at large radii.

end-on, the measured luminosity in a recombination line gives a lower limit to the ionizing luminosity seen by that volume of material. The brightest pixels at each projected radius give the most stringent limits. Even in these locations, the derived luminosity will be an underestimate because of the combined effects of optical depth and covering fraction within the region spanned by each pixel on the object; both quantities are likely to have complex and patchy distributions. Suitably located gas must be present to apply this approach, of course; a strongly flaring AGN would look the same as one without suitable neutral gas around the host system.

Despite these clear limitations, this approach could yield useful information on, for example, the history of AGN luminosity, if the amount of underestimation is comparable for the highest pixels at various radii (if not necessarily between objects). Regions farther from the AGN are physically unlikely

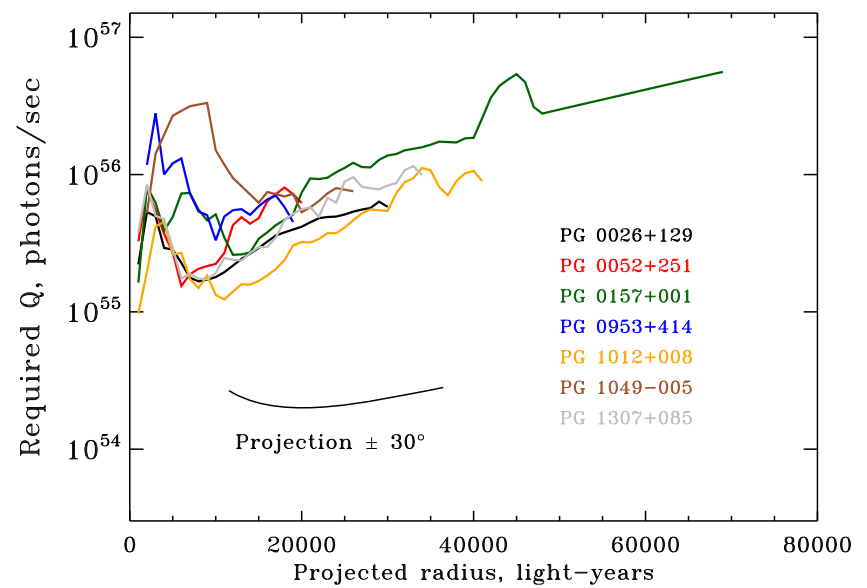

Figure 4. Inferred ionization history of of Palomar-Green QSOs from Bennert et al. (2002), calculated as in Figure 7, showing peak values in bins of 1000 lt$\mathrm{yr}$ with radially adaptive smoothing. The projection curve at the bottom shows the effect of changing the vector direction from the AGN to the gas by $\pm 30^{\circ}$ from the plane of the sky, for a measured value at the bottom of this curve.

to intercept progressively more radiation, since the density in emission regions general falls with distance from the core, and the changes we will infer span orders of magnitude in the opposite sense to what is expected from this. Two immediate checks on this approach are available-the photoionization modeling for spectra of various regions around the Teacup AGN (Gagne et al. 2014), and narrowband [O III] structures of a set of Palomar-Green (PG) QSOs.

We employ the ionization balance as follows. We use the highest surface brightness observed in $\mathrm{H} \alpha$ as a function of projected distance from the AGN to estimate the required (isotropic) emission rate of ionizing photons $Q_{\text {ion }}$ needed to power the observed emission, making the broad assumption as noted above that the densest gas clumps are similar at various projected distances from the AGN within each system. In practice, we use the required photon rate between the $\mathrm{H}$ and $\mathrm{He}$ ionization edges, since the photoionization cross section of helium is large enough to dominate the absorption immediately above $54.6 \mathrm{eV}$. As expected, the HST images reveal numerous small regions of high surface brightness, so that the lower limit we derive in this way is often much higher than estimated from ground-based spectra (Keel et al. 2012a). Our calculation incorporates the fraction of recombinations leading to an $\mathrm{H} \alpha$ photon (Spitzer \& Greenstein 1951; Nussbaumer \& Schmutz 1984). In "Case B," where the gas is optically thick to $\mathrm{Ly} \alpha$, the fraction of recombinations leading to an $\mathrm{H} \alpha$ photon ranges roughly from 0.25 to 0.34 depending on the trapping properties of Ly $\alpha$. The other limit, case A where the nebula is optically thin in $\operatorname{Ly} \alpha$, has these fractions lower by a factor 1.6 (these values are for $T_{e}=10^{4} \mathrm{~K}$, with recombination coefficients as collected by Osterbrock \& Ferland 2006). If we denote the fraction of recombinations leading to an $\mathrm{H} \alpha$ photon by $f, Q_{\text {ion }}$ can be derived conveniently from the areanormalized count rate $N$. This value represents the arrival rate $\left(\mathrm{Hz} \mathrm{cm}^{-2}\right)$ of line photons in the imaging data, corrected for the quantum efficiency $\eta(\approx 0.39$ of the $H S T+\mathrm{ACS}+$ filter system at $\mathrm{H} \alpha$; for NGC 5972, where $\mathrm{H} \alpha$ was observed with WFC3 and the F673N filter, the value is 0.22 ; for NGC 5252 with the WFPC2 ramp filter it is 0.119 ). The current $H S T$ pipeline calibration corrects for the gain (yielding results in electrons per second, $N$, which is to say detected photons) for ACS and WFC3 data, but not for WFPC2 data (so the gain factor must be applied to these images); the incident flux of $\mathrm{H} \alpha$ photons is then $N A / \eta$ for 

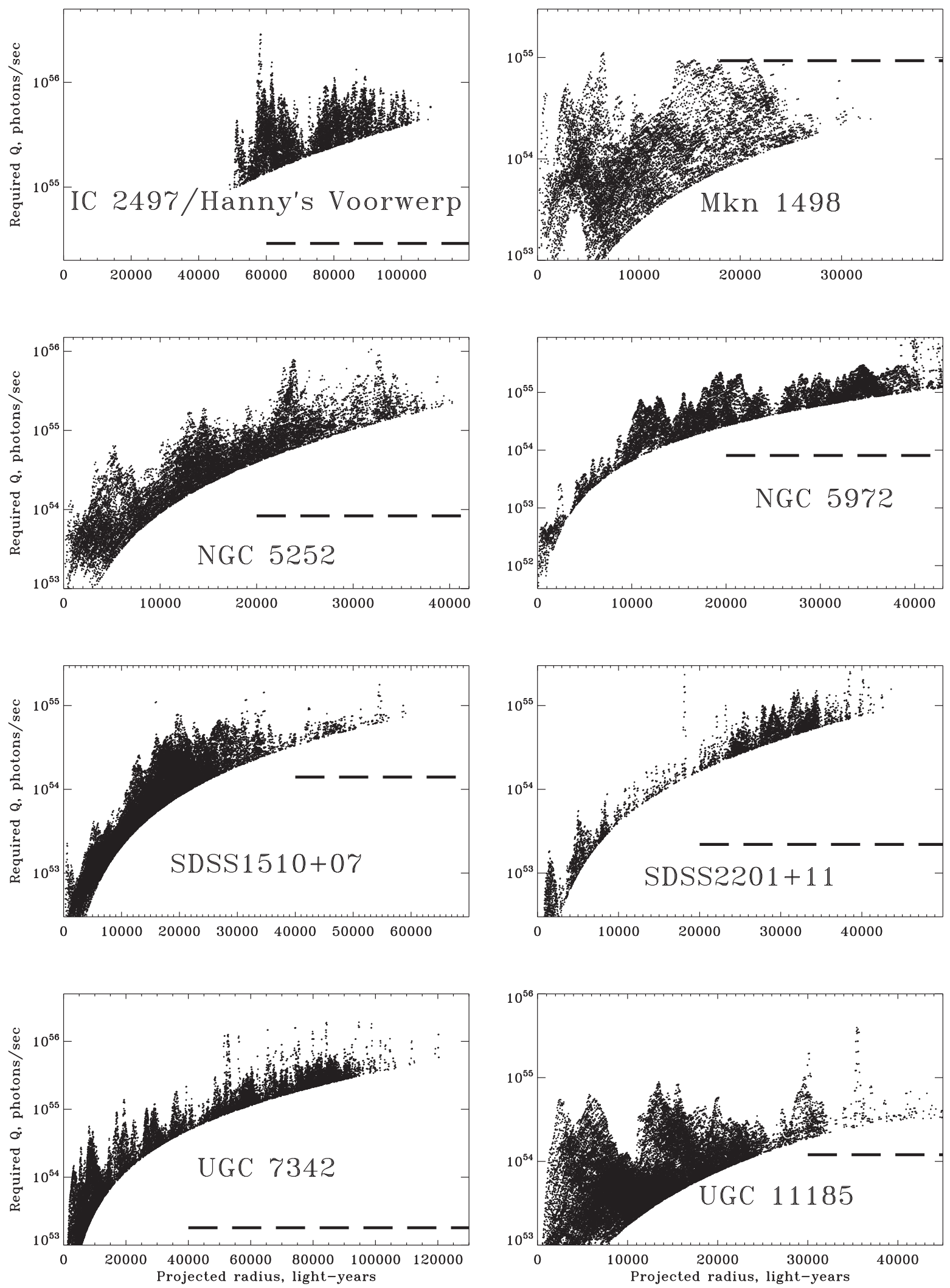

Figure 5. Inferred ionization history of extended AGN-ionized clouds reconstructed pixel by pixel from the surface brightness in H $\alpha$ for each of our sample galaxies, as in Figure 3. The upper envelope is likely to scale with the ionizing luminosity, falling short by a factor related to the optical depth of the material in the brightest pixels (which we assume to be comparable at all radii). Horizontal dashed lines show the ionizing-photon rate inferred from the MIR+FIR luminosity in the upperlimit case of a heavily obscured AGN; the levels shown for SDSS 1510+07 and SDSS 2201+11 are themselves based on IR upper limits. Values above this cannot be accounted for in a simple case of an isotropic AGN heavily obscured along our line of sight. 


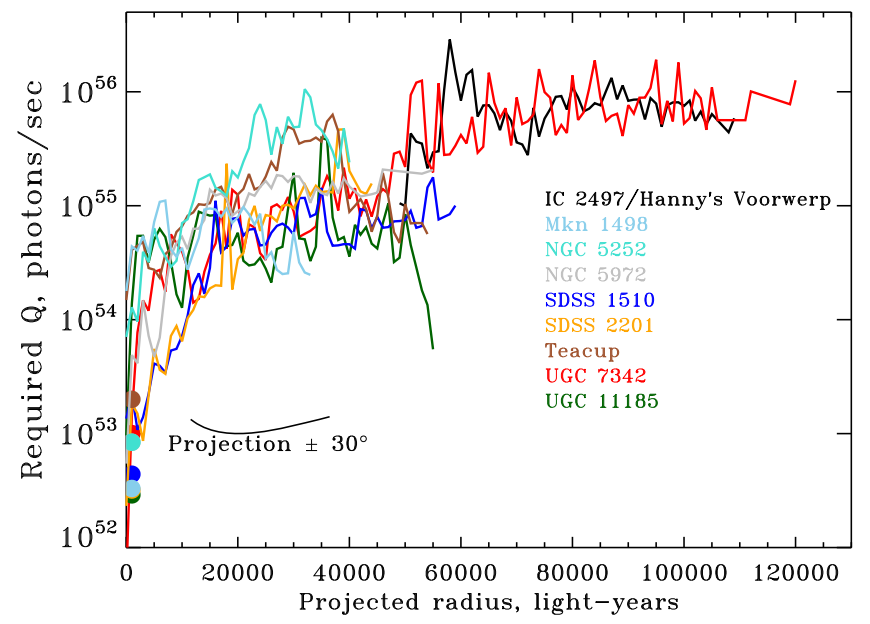

Figure 6. Inferred ionization history of extended AGN-ionized clouds as in Figure 3, with lines showing the peak values in every $1000 \mathrm{lt}-\mathrm{yr}$ bin for each object. The projection curve at the bottom shows the effect of changing the vector direction from the AGN to the gas by $\pm 30^{\circ}$ from the plane of the sky. The large points near zero radius show the derived observed-epoch AGN luminosity from $\mathrm{H} \alpha$ at the nuclei, except for IC 2497, which falls well off the scale at $1.1 \times 10^{50}$ photons $\mathrm{s}^{-1}$.

unobscured telescope area $A$, so the number emitted per second in the emitter's frame is $4 \pi D^{2}(1+z) N A / \eta$ for source distance $D$, now including the time dilation $(1+z)$ in photon arrival rate. Neglecting projection effects, which are poorly known, material in each pixel of observed solid angle $a$ would intercept a fraction of the emitted radiation of the order of $a / 4 \pi R^{2}$ where $R$ is the projected distance from the nucleus to the area of interest; if $R$ is in pixels, $a=1$ for unsmoothed data.

Collecting these components, the count rate $N$ per unit area in a single pixel implies

$$
Q_{\text {ion }}=(1+z)(N / \eta f) 4 \pi D^{2} 4 \pi R^{2}
$$

for a region projected $R$ pixels from the AGN. For the ranges of density and Ly $\alpha$ optical depth that matter here, as noted above, $f$ ranges from 0.16 to 0.34 . This value of $Q_{\text {ion }}$ is a lower limit, since we then have no constraint on how much radiation escapes the system. To be conservative, we discuss values using $f=$ 0.29 , in the middle of the range of Case B photoionization. This was cast in a somewhat different way in Keel et al. (2012a), using the angular width of a spectrograph slit about the nucleus at a given $R$.

The ACS ramp-filter passband has a nearly flat profile near the peak wavelength, falling below $50 \%$ of peak transmission well outside the span of the [N III] lines when centered on $\mathrm{H} \alpha$ at these redshifts. We make a correction for the adjacent [N III] emission based on our slit spectroscopy (Keel et al. 2012a), assuming the behavior of the line ratio to be circularly symmetric about the nuclei on each side. We fit this correction factor as a polynomial in radius for computational convenience.

For optically thin gas, or material with a small covering factor (when shadowing by absorbers closer to the nucleus is not important), there will be an upper envelope in the diagram of $\mathrm{H} \alpha$ surface brightness-projected radius, which will follow a $1 / r^{2}$ form for constant source luminosity as long as various regions in emission do not have radically different projection factors. Departures from this behavior can be evidence of longterm changes in $Q_{\text {ion }}$; neglecting projection factors, one can in principle extract the source history from such a diagram.

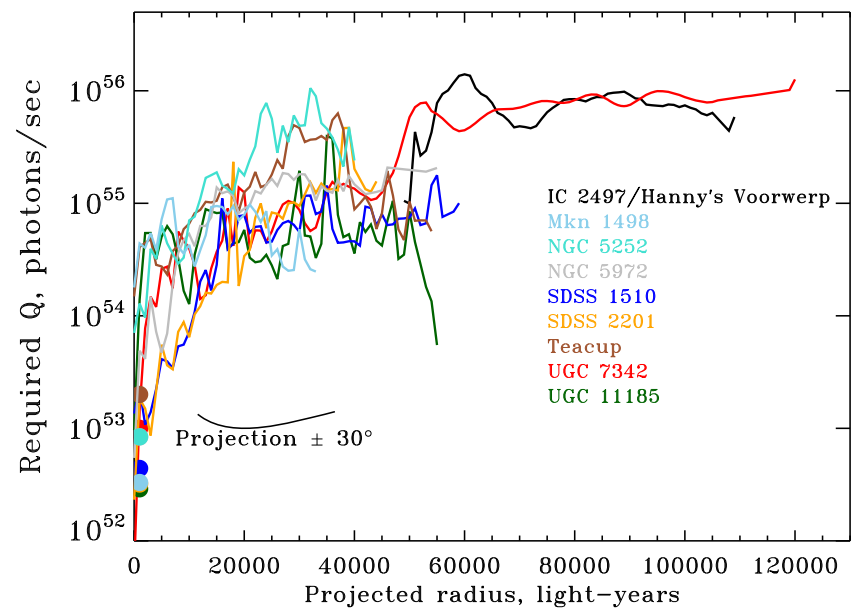

Figure 7. Inferred ionization history of extended AGN-ionized clouds showing the peak values in every 1000 lt-yr bin for each object. This representation smooths the derived $Q$ values by radial amounts intended to reflect changes in the recombination timescale with radius, to better distinguish structure that could be due to AGN variation from gas structure. The projection curve at the bottom shows the effect on a point at the bottom of the curve, of changing the vector direction from the AGN to the gas by $\pm 30^{\circ}$ from the plane of the sky. The large points near zero radius show the derived observed-epoch AGN luminosity from $\mathrm{H} \alpha$ at the nuclei, except for IC 2497, which falls well off the scale at $1.1 \times 10^{50}$ photons $\mathrm{s}^{-1}$.

\subsection{Consistency Tests}

We show one set of data for this calculation suitable for an external test in Figure 2 for the Teacup AGN, where the surface brightness is in electrons pixel ${ }^{-1} \mathrm{~s}^{-1}$ and the radius is in arcseconds. Transforming these into the physically meaningful units of $Q_{\text {ion }}$ and projected distance in light-years gives our estimates of the minimum $Q_{\text {ion }}$ at each pixel.

This conversion is illustrated in Figure 3 , showing $Q_{\text {ion }}$ converted into ionizing luminosity for each pixel above a $3 \sigma$ threshold for the Teacup AGN. The upper envelope is the most significant feature, since we argue that the densest regions are likely to be comparable at various radii. For this object, we can compare our results to the detailed photoionization modeling using the CLOUDY 90 code (Ferland et al. 1998) carried out by Gagne et al. (2014) using ground-based spectra spanning its emission regions. Those results parallel the upper envelope of our reconstructed points, systematically $\approx 25 \%$ higher depending on how the envelope is defined, which supports both our results and the basic assumption of our analysis, that the most optically thick regions are comparable at all radii in the clouds (so we tentatively assume this to be the case for the other galaxies in our sample). In particular, our analysis reproduces the same change in luminosity, declining by a factor $10^{1.03}$ over a time $\Delta T=26,500 \mathrm{yr}$ between the peaks in reconstructed luminosity. It is also reassuring that the peaks in reconstructed luminosity approach the spectroscopic results closely but do not exceed them at any point.

As a second test of our approach, we use HST data on lowredshift QSOs for their high spatial resolution, since numerical tests on our images show that the derived peak ionizing flux drops rapidly with lower image quality. (If this behavior proves to be consistent enough, an analogous study could be done on the emerging body of integral-field spectroscopic mapping of AGNs with EELRs, such as Liu et al. (2013) or Husemann et al. (2016).) We compare with the narrowband [O III] data shown for seven PG QSOs by Bennert et al. (2002), which use 
Table 4

Timescale Estimates for Variability Episodes

\begin{tabular}{|c|c|c|c|c|}
\hline Galaxy & Data Span (yr) & Time Range (yr) & Behavior & $e$-folding Timescale $(\mathrm{yr})$ \\
\hline \multirow[t]{3}{*}{ IC 2497} & 65,000 & $111,000-102,000$ & brighten $1.0 \mathrm{dex}$ & 4000 \\
\hline & & $102,000-60,000$ & constant & $\ldots$ \\
\hline & & $60,000-46,000$ & fade $2.0 \mathrm{dex}$ & 3000 \\
\hline & & $14,000-2000$ & fade 0.5 dex & 10,000 \\
\hline & & $2000-0$ & fade $1.6 \mathrm{dex}$ & 550 \\
\hline NGC 5252 & 40,000 & $40,000-14,000$ & constant & $\ldots$ \\
\hline \multirow[t]{4}{*}{ NGC 5972} & 55,000 & $55,000-15,000$ & fade $0.3 \mathrm{dex}$ & 5800 \\
\hline & & $15,000-5000$ & fade $1.0 \mathrm{dex}$ & 4300 \\
\hline & & $5000-3000$ & brighten $0.5 \mathrm{dex}$ & 1700 \\
\hline & & $3000-0$ & fade $1.3 \mathrm{dex}$ & 1000 \\
\hline \multirow[t]{2}{*}{ SDSS 1510} & 60,000 & $60,000-18,000$ & constant & $\ldots$ \\
\hline & & $18,000-2000$ & fade $1.8 \mathrm{dex}$ & 3900 \\
\hline \multirow[t]{2}{*}{ Teacup } & 55,000 & $55,000-36,000$ & brighten $1.0 \mathrm{dex}$ & 8000 \\
\hline & & $36,000-1000$ & fade $1.2 \mathrm{dex}$ & 12,000 \\
\hline (Gagne et al.) & 39,000 & $39,000-0$ & fade $1.7 \mathrm{dex}$ & 9800 \\
\hline \multirow[t]{6}{*}{ UGC 7342} & 120,000 & $120,000-50,000$ & constant & $\ldots$ \\
\hline & & $50,000-34,000$ & fade $1.03 \mathrm{dex}$ & 6700 \\
\hline & & $34,000-20,000$ & fade $0.1 \mathrm{dex}$ & 60,000 \\
\hline & & $20,000-11,000$ & fade 0.40 dex & 9700 \\
\hline & & $11,000-2000$ & fade 0.6 dex & 6500 \\
\hline & & $2000-0$ & fade $>1.0 \mathrm{dex}$ & 900 \\
\hline \multirow[t]{3}{*}{ UGC 11185} & 55,000 & $55,000-45,000$ & brighten $1.0 \mathrm{dex}$ & 4300 \\
\hline & & $45,000-4000$ & constant & $\ldots$ \\
\hline & & $4000-0$ & fade 2.2 dex & 800 \\
\hline
\end{tabular}

Table 5

Nuclear $\mathrm{H} \alpha$ Fluxes and Ionizing Rates

\begin{tabular}{lccr}
\hline \hline Galaxy & $\begin{array}{c}\text { H } \alpha \text { flux } \\
\left(\mathrm{erg} \mathrm{cm}^{-2} \mathrm{~s}^{-1}\right)\end{array}$ & $\begin{array}{c}Q_{\text {ion }} \\
\left(\text { photons s }^{-1}\right)\end{array}$ & $\begin{array}{c}\text { MIR+FIR } Q_{\text {ion }} \\
\left(\text { photons s }^{-1}\right)\end{array}$ \\
\hline IC 2497 & $1.3 \times 10^{-15}$ & $2.6 \times 10^{50}$ & $2.9 \times 10^{54}$ \\
Mkn 1498 & $5.5 \times 10^{-14}$ & $3.3 \times 10^{52}$ & $9.3 \times 10^{54}$ \\
NGC 5252 & $4.5 \times 10^{-13}$ & $8.4 \times 10^{52}$ & $8.3 \times 10^{53}$ \\
NGC 5972 & $2.6 \times 10^{-14}$ & $8.4 \times 10^{52}$ & $8.1 \times 10^{53}$ \\
SDSS 1510 & $5.9 \times 10^{-14}$ & $4.4 \times 10^{52}$ & $<1.4 \times 10^{54}$ \\
SDSS 2201 & $1.1 \times 10^{-13}$ & $3.2 \times 10^{52}$ & $<2.2 \times 10^{53}$ \\
Teacup & $7.9 \times 10^{-14}$ & $2.0 \times 10^{53}$ & $9.5 \times 10^{54}$ \\
UGC 7342 & $1.3 \times 10^{-13}$ & $1.0 \times 10^{53}$ & $1.8 \times 10^{53}$ \\
UGC 11185 & $4.9 \times 10^{-14}$ & $2.9 \times 10^{52}$ & $1.2 \times 10^{54}$ \\
\hline
\end{tabular}

the WFPC2 linear-ramp filters and continuum subtraction incorporating stellar point-spread functions. The scaling of their implied ionizing luminosities is only approximate; we used a mean value for $[\mathrm{O} \mathrm{III}] / \mathrm{H} \alpha=4$, lacking independent narrow-line ratios at the core. That program was targeted at the sizes of narrow-line regions, with no selection for extent of EELRs, and only three of the PG QSOs have detected emission beyond the $10 \mathrm{kpc}$ radial bound used to select our fading-AGN sample. We masked regions around obvious companion galaxies, but not a bright emission region within $1^{\prime \prime}$ of PG $0157+001$ since its nature is ambiguous. The implied histories are shown in Figure 4. These show brightening, fading, and near-constant episodes. PG $0157+001$ and PG $1012+008$ show fading by nearly an order of magnitude before brightening close to the observed epoch, while PG 0953+414 has brightened by nearly this much. Brightening episodes will be harder to detect at large radii because the signal drops more strongly near the detection threshold. This test broadly confirms that our sample of fading candidates does indeed show distinct behavior using our technique of reconstructing luminosity from recombination balance. In particular, none of the PG QSOs shows the rapid drops in the last 20,000 yr that are ubiquitous in our fading-AGN sample.

\subsection{Luminosity Histories of Fading AGNs}

The two external tests outlined above suggest that, indeed, we can reconstruct at least qualitative histories for AGN luminosity using pixel-by-pixel recombination balance. Figure 5 shows these pixel-by-pixel results for the other seven objects in our sample plus IC 2497 and Hanny's Voorwerp, where similar data were described by Keel et al. (2012b). Figure 6 compares the upper envelopes for all objects, connecting the brightest pixels in bins of 1000 lt-yr.

Recombination timescales for hydrogen approach $10^{4} \mathrm{yr}$ in the outer parts of these clouds, so only changes on longer terms can reflect variations in the AGN output; along with line-ofsight depth of the cloud systems, this means that the temporal resolution degrades with projected radius. We illustrate this in Figure 7, which smooths the derived luminosities by a range 0.14 times the radius, a value that matches the best-constrained timescale in the outer regions of Hanny's Voorwerp and 


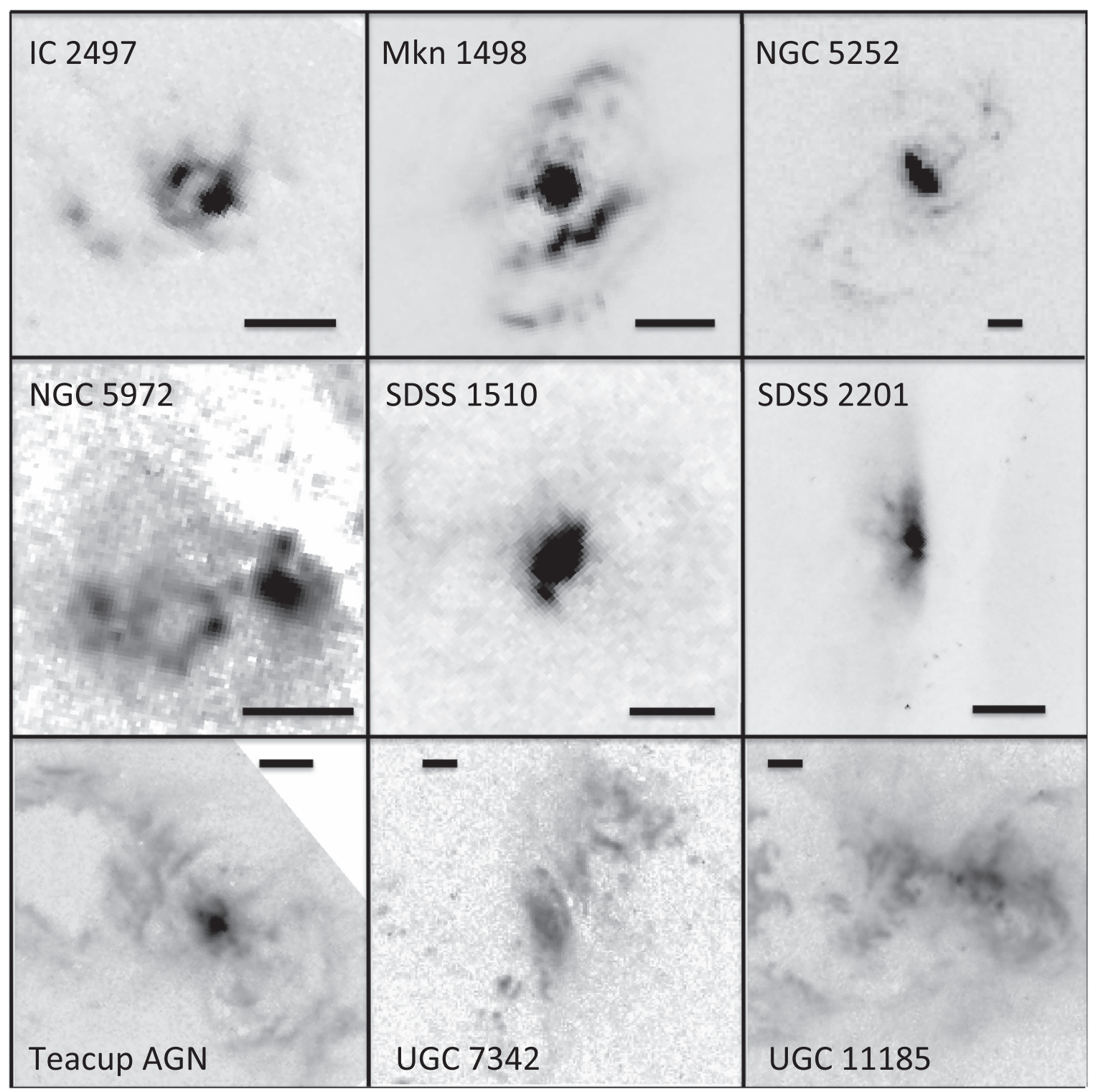

Figure 8. Continuum-subtracted $\mathrm{H} \alpha+[\mathrm{N}$ III] images of nuclear regions, most showing loops or ringlike emission structures suggesting outflow. In NGC 5972 and SDSS 2201, color changes from dust lanes limit the precision of continuum subtraction with the available filters. North is at the top, east to the left; scale bars are $1^{\prime \prime}$ long. The three objects in the bottom row have very bright central emission, so their images are shown with logarithmic intensity scales; the rest use linear scales.

roughly accounts for a decrease in density with radius matching the limits from Lintott et al. (2009). The points at $\Delta T=0$ in both versions of this figure are from the $\mathrm{H} \alpha$ fluxes at the nuclei (operationally within a projected radius of $1^{\prime \prime}$ from slit spectroscopy), in the approximation that they represent full covering of the instantaneous ionizing flux (strictly, a lower limit since we see that ionizing photons do escape the nuclear region). The flux values are taken from our spectroscopy in Keel et al. (2015), as given in Table 5, including only the narrow component of the composite profile in Mkn 1498 for consistency. As a rough numerical description of these reconstructed histories, we collect in Table 4 a set of properties fit exponentially to segments of the smoothed version in Figure 7, showing not only fading periods but constant and brightening episodes.

We considered several alternative approaches to calculating ionizing history, including integrating the line flux in slices across the putative ionization cones. This was defeated by the very patchy structure of the ionized features; tracing only the peak intensity reduces sensitivity to the larger-scale structure.

We can estimate the values of $Q_{\text {ion }}$ predicted from the complementary assumption that the AGN is unseen along the direct line of sight due to dust obscuration. In this case, we take the total infrared output to be the total AGN luminosity, and a typical fraction of the bolometric luminosity 0.16 to be in ionizing radiation from 13.6 to $54 \mathrm{eV}$ (typical of composite spectral energy distributions, although this is often the least well-determined piece of the spectrum). For a wide range of spectral index in this range, the mean ionizing energy is close to $27 \mathrm{eV}$ (Netzer 1990, p. 57). This is intended to be conservative with respect to the possible role of AGN observation, making no correction for host-galaxy IR contribution or the possibility that significant IR reprocessing occurs far from the AGN and thus may not reflect its luminosity at the same epoch as our direct view. The resulting $Q_{\text {ion }}$ values or 

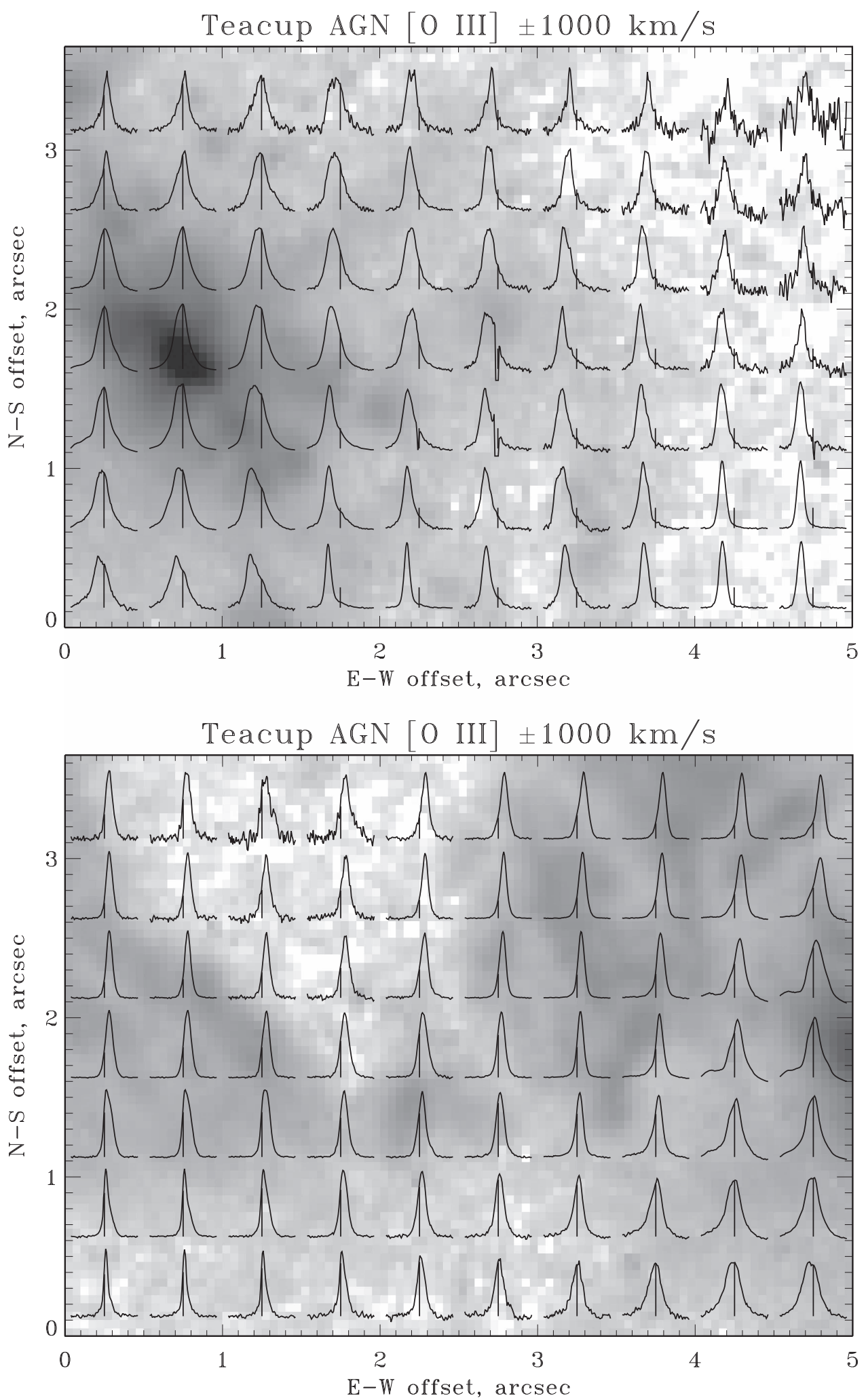

Figure 9. [O III] emission-line profiles from the GMOS IFU spectra overlaid on the HST [O III] images, shown with a common logarithmic intensity scale. Line profiles are from the data averaged over $0.15 \times 0 . ! 5$ regions, and are scaled to have the same peak heights. The velocity range shown is indicated at the top of each panel- $\pm 700 \mathrm{~km} \mathrm{~s}^{-1}$ for all but the Teacup, where we show $\pm 1000 \mathrm{~km} \mathrm{~s}^{-1}$. The coordinates are in the GMOS IFU field system for ease of display; this involves $90^{\circ}$ rotations between some of them, and the UGC 7342 regions have south at the top. Alignment was set by comparison of smoothed versions of the HST images to monochromatic slices of the IFU data cubes. For the three Teacup AGN pointings, the first includes the AGN itself, and the next two include the southern and northern parts of the prominent loop to its east. For each line profile, the vertical line marks the systemic velocity as derived from the nucleus. The Gemini data have excellent sensitivity to diffuse emission, giving detection of emission even in some areas that are essentially blank in the HST images. 

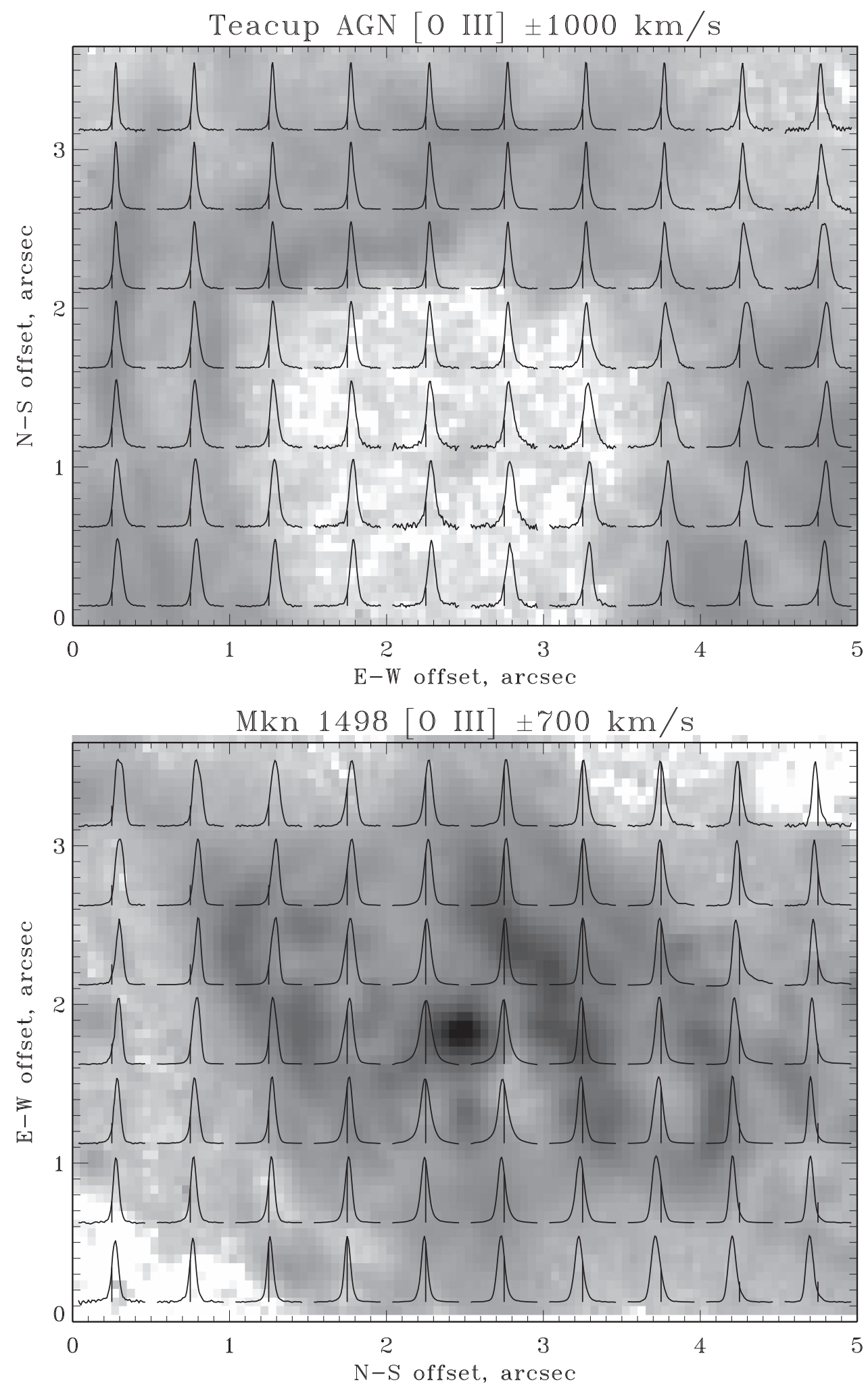

Figure 9. (Continued.)

limits for most of these AGNs (listed in Table 5) fall well below even the lower limits imposed by recombination balance, adding to the case for drops in luminosity. Finally, the spatially resolved drops in required ionizing flux seen within each object's clouds add to the evidence that the energy mismatch between nuclei and extended clouds really results from variability rather than unusually hard FUV spectral slopes or localized extinction around the AGN. The shortfalls for $Q_{\text {ion }}$ derived from the infrared are less extreme, indicating modest local obscuration, but in most cases still very large.

Most of our sample would not have shown enough fading to satisfy our selection criteria based only on the early parts of the 

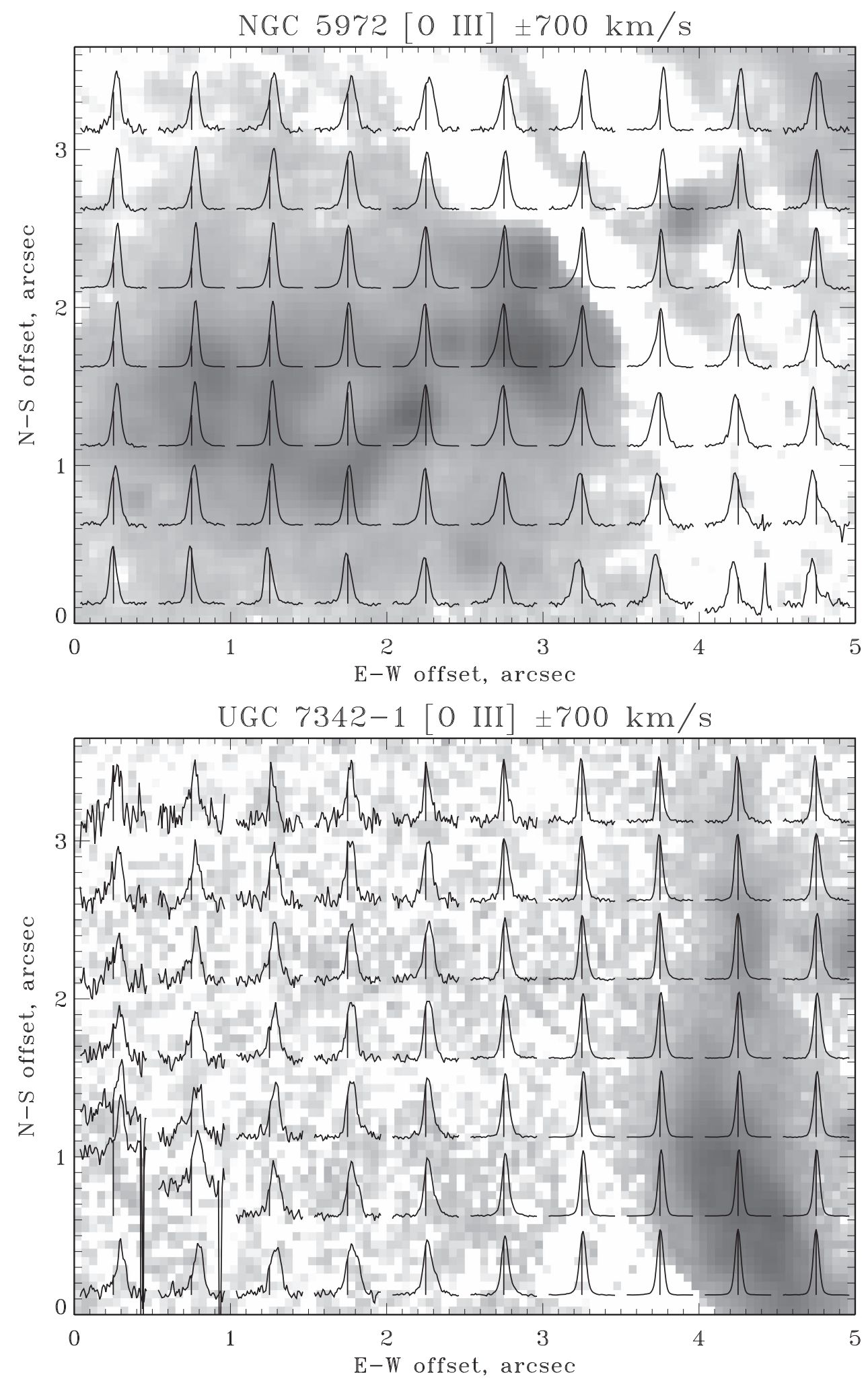

Figure 9. (Continued.)

reconstructed histories. The strongest, characteristic fading took place within the last 20,000 yr before the epoch when we observe the nuclei, except for IC 2497/Hanny's Voorwerp, where it occurred in a similar span about 50,000 yr before the direct view and remained dim. This distinctive fading is evidence (internal to each galaxy) for actual drops in luminosity rather than alternative interpretations of the mismatch in energy budget in these objects, such as obscuration from dust far from the nucleus (so there is no necessary connection between the obscuration and mid-IR emission) or spectral energy distributions unusually strongly peaked in the ionizing UV. 


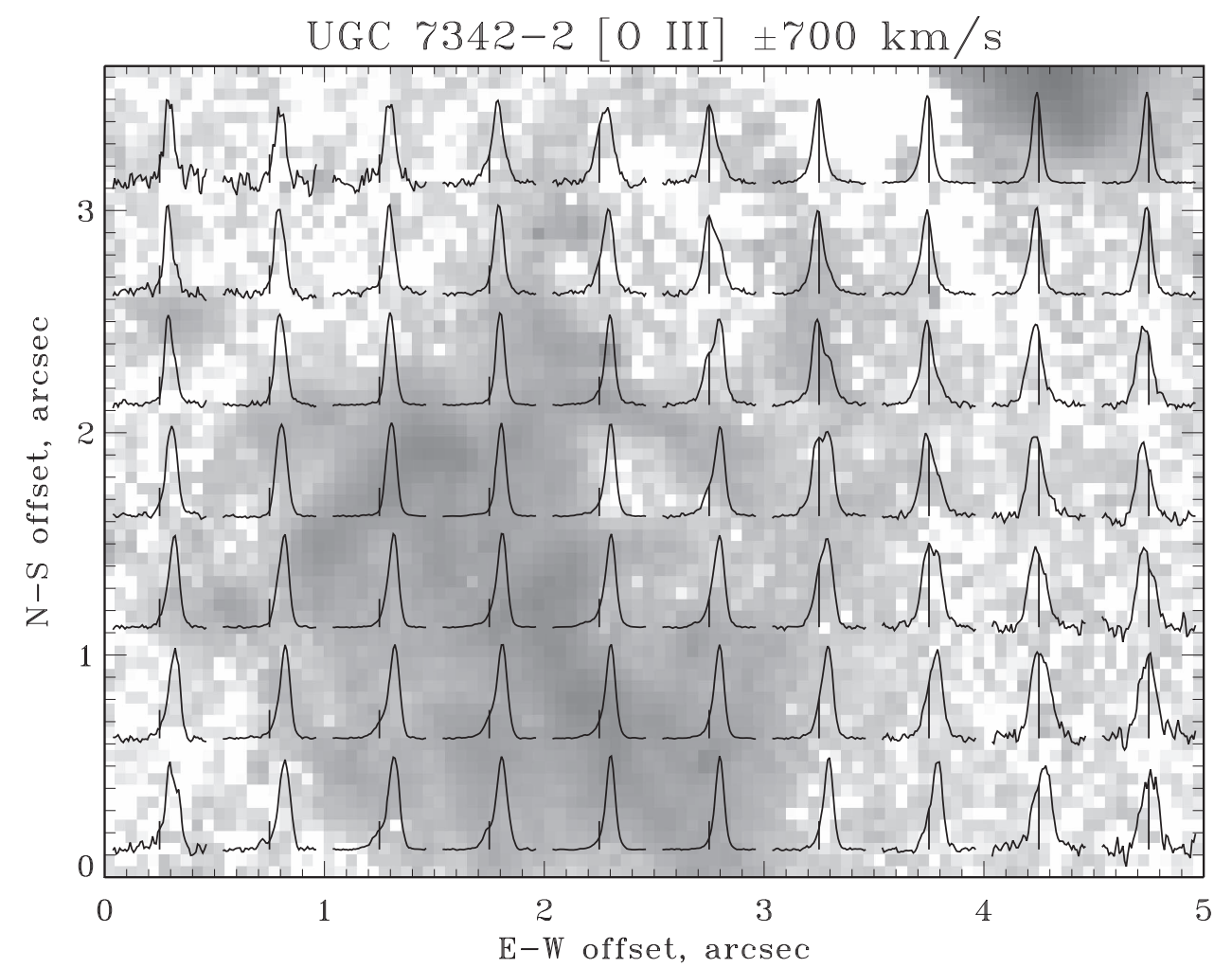

Figure 9. (Continued.)

\section{AGN Outflows and Feedback}

In IC 2497, host galaxy of Hanny's Voorwerp, HST observations reveal an expanding loop of low-ionization gas extending $\approx 500 \mathrm{pc}$ to one side of the nucleus (Keel et al. 2012b). This, along with evidence for an oppositely directed radio jet (Józsa et al. 2009; Rampadarath et al. 2010) and bubble-like structure in the X-ray gas (Sartori et al. 2016), invites speculation that these objects are not necessarily undergoing a near-shutdown of accretion, but may (also) be switching modes of energy output incidental to the accretion, to become kinematically dominated (sometimes known as radiomode). In X-ray binaries, the switch from radiative to kinetic mode is associated with a change in accretion state (and thus a decrease in the luminosity). It is possible that the same is happening also in AGNs (Sartori et al. 2016). There are additional AGNs with well-studied bubbles or loops attributed to outflows, such as recent results on NGC 3393 by Maksym et al. (2017). This motivated us to look for similar loops of emission, and seek kinematic evidence as to whether they represent outflows or something else.

Morphologically similar loops to one side of nuclei, or encircling them, are common in this sample (Figure 8), and could by themselves be signatures of outflow episodes. However, the GMOS IFU data give a more complex picture both kinematically and in emission-line ratios.

Kinematic signs of outflow do appear in several nuclei, although some of the loops are more nearly in rotation than radial motion. We can evaluate this using the Gemini IFU spectroscopy, as well as the wider-field Fabry-Perot velocity fields for [O III] obtained with the $6 \mathrm{~m}$ BTA (Paper I). Spectroscopic signatures of outflow include multiple-peaked or asymmetric line profiles, and departures from large-scale rotational velocity fields even for locally narrow and symmetric profiles. Such features are seen in confined regions of some of our target galaxies. Figure 9 overlays the [O III] profiles for the Gemini IFU data on HST narrowband images in [O III], with the line profiles representing averages of the data over $0.5 \times 0$." 5 regions.

In Mkn 1498 the ringlike emission features that are prominent at $\mathrm{H} \alpha$ are dominated kinematically by rotation, possibly with one outflowing feature adjacent to the nucleus (Figure 10). The implied rotation axis is close to the minor axis of the largest emission-line structures, making it almost parallel to the large-scale radio structure. In the line profiles, red wings occur along the outer ring to the south, and about $2^{\prime \prime}$ east of the nucleus along the other ring (but not the rest of it). The inner arc structure to the northwest of the nucleus has narrow line profiles, but departs from the kinematic pattern of the velocity peaks elsewhere in the ring.

As seen in Figure 9, UGC 7342 shows substantial regions with double emission-line peaks, likely evidence of outflow in at least one component. We examine this in more detail by fitting sets of two Gaussian [O III] profiles where this improves the fit, constraining them to have equal widths for consistency across a range of $\mathrm{S} /$ Ns. Similar behavior is seen in $\mathrm{H} \alpha$ and $\mathrm{H} \beta$, but $\mathrm{H} \beta$ is weaker and $\mathrm{H} \alpha$ is affected by overlap with [N III] components. The results are shown in Figure 11. Only peak separations $>100 \mathrm{~km} \mathrm{~s}^{-1}$ are detected, in view of the typical line width of $230 \mathrm{~km} \mathrm{~s}^{-1}$ FWHM. Component separations range up to $270 \mathrm{~km} \mathrm{~s}^{-1}$. The pattern of component velocities is not a simple two-sided outflow, and may have more than one origin. The outer part of the filamentary emission region about 3 ." 5 northeast of the nucleus shows velocity splitting. This cloud hosts the most intense emission seen in the redder component (whose radial velocity changes from place to place), while its outer part shows additional blue wings (Figure 9). In addition, perpendicular to 

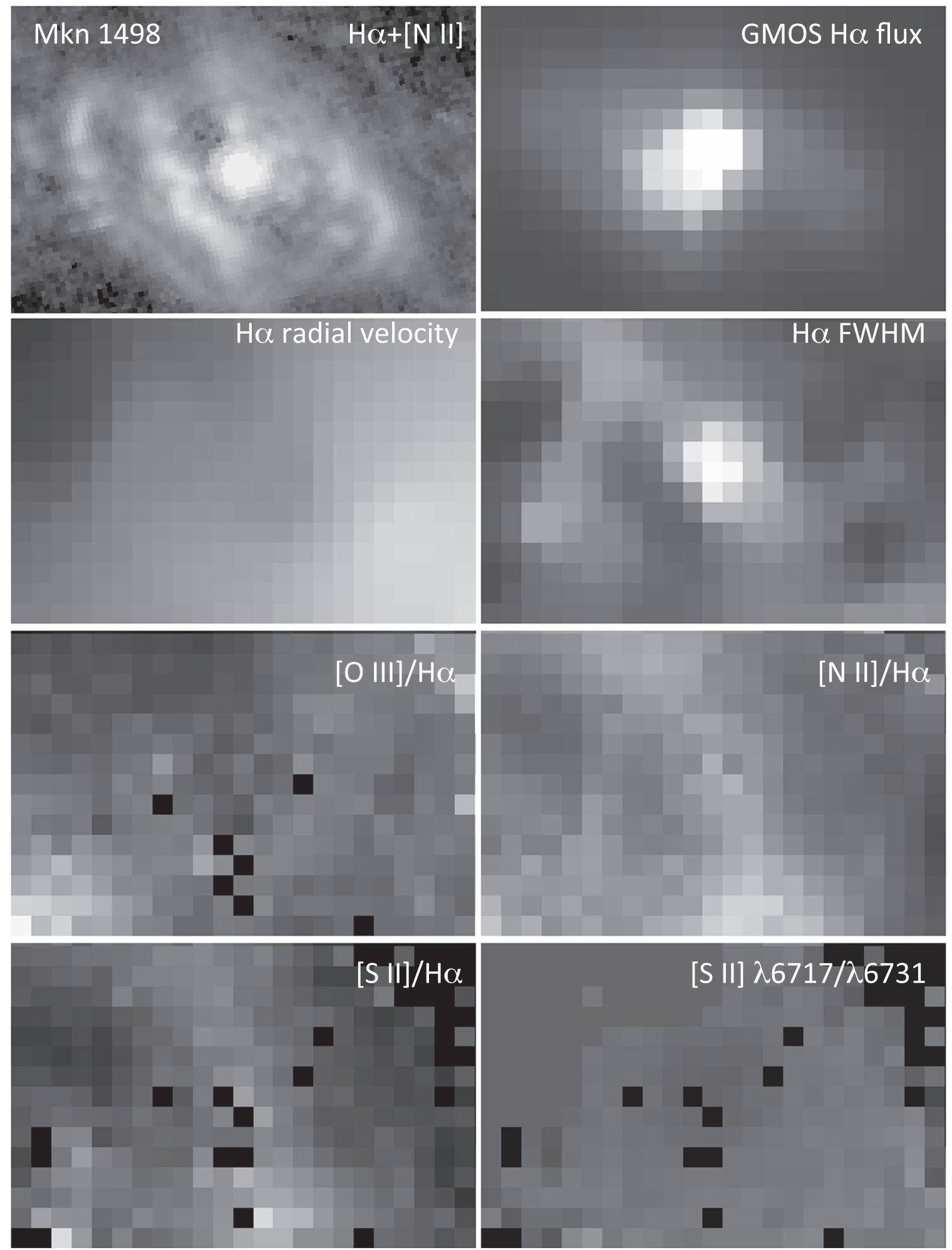

Figure 10. Summary of GMOS IFU results in the inner $3.4 \times 4.8$ arcsec of Mkn 1498. North is on the right, east at the top. The HST narrowband image includes both $\mathrm{H} \alpha$ and $[\mathrm{N} \mathrm{III]}$ lines. For all quantities, lighter shades represent larger values.

the main axis of the emission regions, there are extensive regions with split line profiles; much of this emission is too diffuse to be well detected in the $H S T$ images.

Using the BTA Fabry-Perot global velocity map from Paper I as a guide to the overall velocity field, which is well fitted by a rotating and slightly warped disk, we find that the redder component is the more quiescent one to the south of the nucleus, while the bluer component fills this role to its north and northwest (where large velocity splittings occur in diffuse gas). East of the nucleus, the HST images do show a faint filament that could be associated with one of these velocity structures. The areas with large local velocity dispersion $\sigma_{v}$ in 

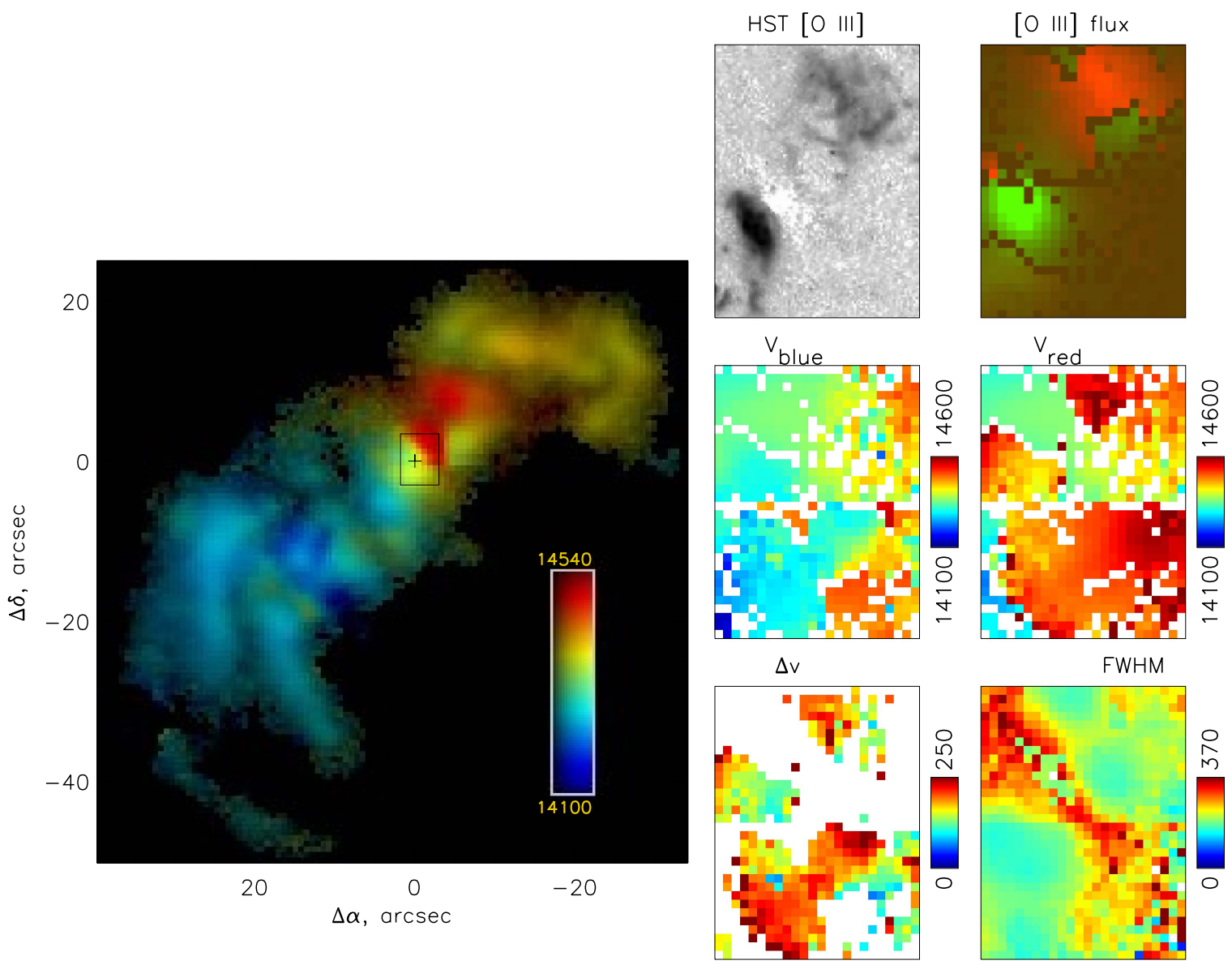

Figure 11. Results of two-component Gaussian-blend fits to [O III] emission in UGC 7342. The HST ACS [O III] image is scaled logarithmically to more fully display the structures near the core. The two GMOS IFU fields are abutted at their nominal locations here, giving contiguous coverage over 4 ." $8 \times 6$ "! 4 . North is at the top, east to the left. The intensities of red and blue (shown in green for contrast) components are scaled independently for better visibility. Some regions of large FWHM are adjacent to double-peaked areas, presumably representing unresolved multiple components. For comparison, the left panel shows the Fabry-Perot velocity field of the entire galaxy as measured with the BTA (Keel et al. 2015), combining hue for radial velocity with luminance for [O III] flux.

the BTA data just to the north of the nucleus are a good match to regions of line splitting in the GMOS data cubes.

We are left with a complex empirical picture for kinematics in the inner few kiloparsecs of UGC 7342. Emission-line components suggest outflow, or at least motions departing from the overall rotation pattern by as much as $260 \mathrm{~km} \mathrm{~s}^{-1}$, in regions of diffuse, low-surface-brightness emission perpendicular to the main emission regions, with the strongest offrotational emission in the outer parts of a bright cloud, part of which also shows blue wings beyond a simple two-component line profile. We might speculate that this could be entrainment of denser gas by a low-density outflow such as seen west of the nucleus. Curiously, the brightest [O III] structure, resembling an inclined ring just southwest of the AGN position, has no distinct signature in radial velocity.

The Teacup AGN was shown to have resolved outflow signatures by Harrison et al. (2015). We find double or asymmetric profiles extending $2^{\prime \prime}$ north, $4^{\prime \prime}$ west, $>1$ ". 5 south, and $2^{\prime \prime}$ east in a roughly circular zone. What appears as an [O III] peak in the center of the loop on the underlying HST image may be affected by residuals from cosmic-ray events. The Gemini data are better at detecting emission that is diffuse and at low surface brightness, as in the center of the loop, and show no distinct emission-line component there. Blue wings dominate east of the nucleus, red wings to the west; in many locations they extend to $1000 \mathrm{~km} \mathrm{~s}^{-1}$ from the systemic velocity.

NGC 5972 shows significant velocity structure, and a nearly constant ionization level (Figure 12). It shows double and asymmetric peaks near the dust lane, which can occur strictly from obscuration in a continuous velocity field, and in the inner part of the emission-line loop to its east. Overall, the emissionline loop to the east of the nucleus shows no discernible velocity signature; it does have narrower line profiles than its surroundings, perhaps because its stronger emission dominates the more diffuse gas elsewhere along the line of sight. There is larger-scale velocity structure in the GMOS field spanning $\approx 100 \mathrm{~km} \mathrm{~s}^{-1}$, not matching the loop morphology or location; this is part of the departures from a fit to circular motions seen near the nucleus in the wide-field BTA Fabry-Perot map (Keel et al. 2015). The [O III $] / \mathrm{H} \alpha$ ratio declines with radius from the nucleus at the same rate in the loop and its surroundings; the loop does not stand out in this parameter. It has lower [N III]/ $\mathrm{H} \alpha$ and $[\mathrm{S} \mathrm{II}] / \mathrm{H} \alpha$ ratios than its surroundings, but not [O III]/ $\mathrm{H} \alpha$ or the density-sensitive [S II] $\lambda 6717 / \lambda 6731$ ratio. The loop region 2 " 0 east of the nucleus has $[\mathrm{N}$ III] $\lambda 6583 / \mathrm{H} \alpha=0.33$ $(0.71$ in the surroundings) and ([S II] $\lambda \lambda 6717+6731) /$ $\mathrm{H} \alpha=0.36$ (0.70). Both line ratios are more sensitive to abundances than to ionization parameter under AGN-like photoionization, unlike $[\mathrm{O} \mathrm{III}] / \mathrm{H} \alpha$ (Storchi-Bergmann et al. 


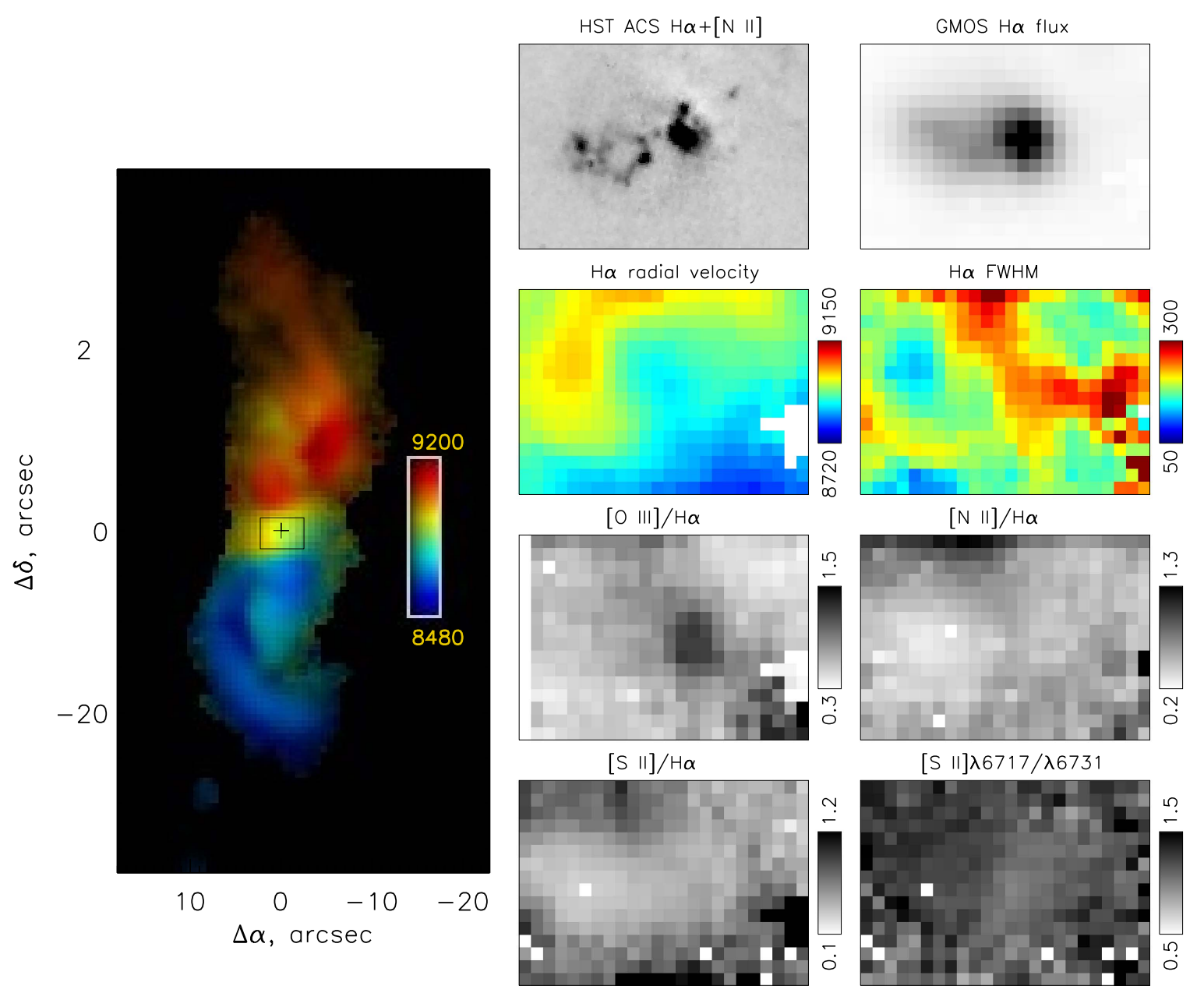

Figure 12. Summary of GMOS IFU results in the inner $3 "$ " $4 \times 4$ " 8 of NGC 5972. North is at the top, east to the left. The HST narrowband image includes both H $\alpha$ and [N III] lines. The peak FWHM of $326 \mathrm{~km} \mathrm{~s}^{-1}$ occurs 0 "! 2 east of the peak $\mathrm{H} \alpha$ flux. For comparison, the left panel shows the Fabry-Perot velocity field of the entire galaxy as measured with the BTA (Keel et al. 2015), combining hue for radial velocity with luminance for [O III] flux.

1998). Very broadly, the line ratios show lower abundances in this small-scale loop of emission than in its surroundings, which could suggest, contrary to its appearance, that it consists of infalling or recently accreted gas. Together, these properties seem more like infall than an AGN-driven outflow; the alternative would require a fairly contrived set of circumstances, such as motion in the plane of the sky and the outflow itself being unseen (for example, too hot for optical emission) and entraining material that originated in a low-luminosity companion disrupted by the obvious merger history. Young stars might give the observed line ratios, in which case it would be a coincidence that the $[\mathrm{O} \mathrm{III}] / \mathrm{H} \alpha$ ratio matches the surrounding AGN-ionized gas so closely, but no luminous star clusters appear in WFC3 continuum images (Keel et al. 2015).

\section{Comparison: Changes in Luminosity in Accretion Simulations}

We can compare the changes in luminosity we measure to simulations such as Novak et al. (2011) to see how common such dramatic changes are predicted to be for various feedback properties. Existing simulations have time resolution of the order of $10^{4} \mathrm{yr}$, so there may be just enough information to tell what behavior is expected on timescales close to $10^{5} \mathrm{yr}$. Figure 13 shows how common various levels of drops in accretion luminosity (derived from the simulations as $L / L_{\mathrm{Edd}}$ ) are predicted to be when sampled on various timescales. We emulate the measurements by smoothing the starting luminosity at each time step by $30 \%$ of the timespan being sampled, which mimics the effects of finite depth along the line of sight and increased recombination timescale at the typically lower densities farther from the AGN. Although the time resolution is not really adequate to test what happens when sampling a few $10^{4} \mathrm{yr}$, it is encouraging that this simulation shows a few per cent of its time steps that would satisfy our selection criteria for AGN fading if suitable surrounding gas were present (Table 6). The table lists the fraction of time steps where the luminosity drops at least as steeply as the listed ratios, evaluated for various values of $\Delta T$ according to $L(T) / \overline{L(T-\Delta T)}$ where the averaging in the denominator is over $0.3 \Delta T$ as above. As Novak et al. (2011) note, their simulations show long periods of near-constant accretion, and other periods showing many cycles of nearly periodic changes.

Some simulations indicate that smaller linear scales for changes in the inflow rate (from clumpy accretion or changes 


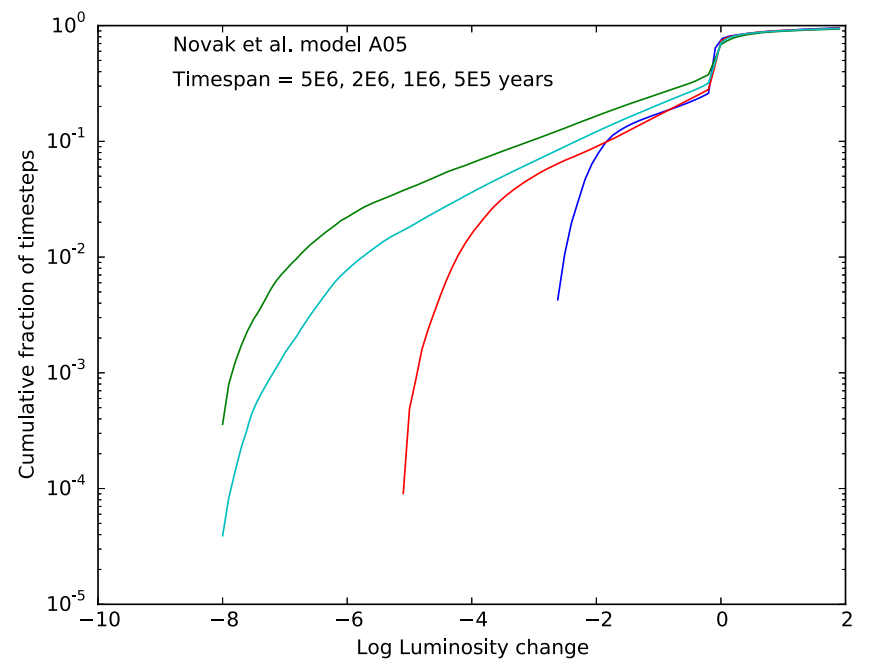

Figure 13. Fraction of time steps from simulation A05 in Novak et al. (2011) as a function of the drop in luminosity $L(T) / \overline{L(T-\Delta T)}$, evaluated on timescales from $5 \times 10^{5}$ to $5 \times 10^{6}$ yr. For each time series, the starting luminosity was smoothed over $30 \%$ of the relevant timespan to roughly model the effects of finite cloud depth along the line of sight and longer recombination times at low densities. The lack of points to the lower right indicates effects of time resolution; at longer timescales we may be seeing effects of physically driven timescales in the simulation.

due to feedback from the AGN) yield faster changes in accretion rate (Gabor \& Bournaud 2013).

\section{Summary}

We present $H S T$ narrowband imaging and Gemini integralfield optical spectra for a set of fading AGNs, selected for shortfalls in the energy budget between giant ionized clouds and the nuclei themselves, suggesting significant reduction in ionizing photons within the last $\approx 2 \times 10^{4} \mathrm{yr}$. In particular, we use recombination balance to estimate the history of radiative output, finding that the feature in common is a radial drop in luminosity within 20,000 $\mathrm{yr}$ before our direct view of the nucleus. For earlier times, we see a range of behaviorsconstant, brightening, fading-which are echoed in similarly reconstructed histories of PG QSOs where [O III] HST imaging is available.

In contrast to many of the extended emission clouds around radio-loud AGNs, these are rotationally dominated and show only very localized outflow velocities greater than $\approx 100 \mathrm{~km} \mathrm{~s}^{-1}$. These EELRs are mostly externally illuminated tidal debris rather than wind material (Keel et al. 2015).

As in Keel et al. (2012a), these results continue to support the idea that AGNs with extended emission regions are bright for periods of $10^{4}-10^{5} \mathrm{yr}$ at a time, with substantially fainter episodes interspersed (which fits with the idea that the opposite behavior-brightening — can be seen in the number of X-raybright AGNs without substantial narrow-line regions; Schawinski et al. 2015). Most galaxies do not have large reservoirs of (extraplanar) cold gas to show such behavior, so we can trace it preferentially in interacting or merging systems where warped disk gas or tidal tails provide a screen to be ionized by escaping ionizing photons.

Among EELR hosts from the Galaxy Zoo sample, fading AGNs make up $\approx 40 \%$ of the total, leading to the simple estimate of long accretion timescales in Keel et al. (2012a). This leaves open the possibility that fading cases could be a specific subset of the whole population. For example, inspiral
Table 6

Simulated Time Fractions in Low States

\begin{tabular}{lcccc}
\hline \hline $\begin{array}{l}\text { Log(Luminos- } \\
\text { ity Drop) }\end{array}$ & $\begin{array}{c}\text { Timespan } \\
5 \times 10^{6} \mathrm{yr}\end{array}$ & $\begin{array}{c}\text { Timespan } \\
2 \times 10^{6} \mathrm{yr}\end{array}$ & $\begin{array}{c}\text { Timespan } \\
10^{6} \mathrm{yr}\end{array}$ & $\begin{array}{c}\text { Timespan } \\
5 \times 10^{5} \mathrm{yr}\end{array}$ \\
\hline-1 & 0.26 & 0.20 & 0.16 & 0.16 \\
-2 & 0.15 & 0.12 & 0.085 & 0.063 \\
-3 & 0.098 & 0.067 & 0.050 & $\cdots$ \\
-4 & 0.063 & 0.037 & 0.015 & $\ldots$ \\
-5 & 0.039 & 0.019 & 0.0005 & $\cdots$ \\
\hline
\end{tabular}

Note. Fraction of time steps in the simulation where the measured luminosity drop with respect to the averaged past value is more extreme than the reference value for each column.

of bound binary supermassive black holes could disturb a preexisting accretion disk, while significant changes in the direction of ionizing radiation could also result from the kinds of tilted disk discussed by Lawrence \& Elvis (2010). In that case, shadowing by the disk itself would leave ionization "cone" shapes that are set by the intersection of two cones of different center directions and opening angles.

This work was supported by NASA through STScI grants HST-GO-12525.01-A and -B. Some of the data presented in this paper were obtained from the Mikulski Archive for Space Telescopes (MAST). This research has made use of NASA's Astrophysics Data System, and the NASA/IPAC Extragalactic Database (NED), which is operated by the Jet Propulsion Laboratory, California Institute of Technology, under contract with the National Aeronautics and Space Administration. We thank Linda Dressel for advice on setting up the HST observations, especially reducing intrusive reflections. Identification of this galaxy sample was possible through the efforts of nearly 200 Galaxy Zoo volunteers; we are grateful for their contributions, and thank once more the list of participants in Keel et al. (2012a). This work was partly supported by a grant from the President of the Russian Federation (MD-3623.2015.2). The observations obtained with the $6 \mathrm{~m}$ telescope of the SAO of the RAS were carried out with the financial support of the Ministry of Education and Science of the Russian Federation (contracts no. 16.518.11.7073 and 14.518.11.7070). We benefited from conversations with Hai Fu, Larry Rudnick, Kelly Holley-Bockelmann, Tamara Bogdanovic, and Stephanie Juneau. Greg Novak kindly provided detailed results of his numerical simulations. We thank Dara Norman and Kathy Roth for key help in setting up the Gemini observations, James Turner for sharing experience in subtleties of their processing, and Gemini staff observers M. Hoenig, A.-N. Chene, J. Chavez, M. Pohlen, L. Fuhrman, and J. Ball for obtaining these data in queue mode.

C. J. Lintott acknowledges funding from The Leverhulme Trust and the STFC Science in Society Program. W.P.M. is grateful for support by the University of Alabama Research Stimulation Program. V.N.B. acknowledges assistance from a National Science Foundation (NSF) Research at Undergraduate Institutions (RUI) grant AST-1312296. Note that findings and conclusions do not necessarily represent views of the NSF. A.M. is also grateful for the financial support of the "Dynasty" Foundation. K.S. was supported by a NASA Einstein Fellowship at Yale, and gratefully acknowledges support from Swiss National Science Foundation Grant PP00P2 138979/1. Galaxy 
Zoo was made possible by funding from the Jim Gray Research Fund from Microsoft and The Leverhulme Trust.

This publication makes use of data products from the Widefield Infrared Survey Explorer, which is a joint project of the University of California, Los Angeles, and the Jet Propulsion Laboratory/California Institute of Technology, funded by the National Aeronautics and Space Administration.

Facilities: HST (ACS, WFC3, WFPC2), BTA, Gemini Gillette, WISE.

\section{References}

Allington-Smith, J., Murray, G., Content, R., et al. 2002, PASP, 114, 892 Bennert, N., Falcke, H., Schulz, H., Wilson, A. S., \& Wills, B. J. 2002, ApJL, 574, L105

Bland-Hawthorn, J., Maloney, P. R., Sutherland, R. S., \& Madsen, G. J. 2013, ApJ, 778, 58

Cutri, R. M., Wright, E. L., Conrow, T., et al. 2014, Explanatory Supplement to the WISE, Section 4.4, All-Sky Data Release Products, http://wise2. ipac.caltech.edu/docs/release/allsky/expsup/sec4_4h.html

Davies, R. L., Allington-Smith, J. R., Bettess, P., et al. 1997, Proc. SPIE, 2871, 1099

Davies, R. L., Schirmer, M., \& Turner, J. E. H. 2015, MNRAS, 449, 1731

Ferland, G. J., Korista, K. T., Verner, D. A., et al. 1998, PASP, 110, 761

Fullmer, L., \& Lonsdale, C. J. 1989, Cataloged Galaxies and Quasars Observed in the IRAS Survey, JPL D-1932

Gabor, J. M., \& Bournaud, F. 2013, MNRAS, 434, 606

Gagne, J. P., Crenshaw, D. M., Kraemer, S. B., et al. 2014, ApJ, 792, 72

Guo, F., \& Mathews, W. G. 2012, ApJ, 756, 181

Hainline, K. N., Hickox, R. C., Chen, C.-T., et al. 2016, ApJ, 823, 42

Harrison, C. M., Thomson, A. P., Alexander, D. M., et al. 2015, ApJ, 800, 45

Husemann, B., Bennert, V. N., Scharwächter, J., Woo, J.-H., \& Choudhury, O. S. 2016, MNRAS, 455, 1905

Józsa, G. I. G., Garrett, M. A., Oosterloo, T. A., et al. 2009, A\&A, 500, L33

Keel, W. C., Chojnowski, S. D., Bennert, V. N., et al. 2012a, MNRAS, 420, 878

Keel, W. C., Lintott, C. J., Schawinski, K., et al. 2012b, AJ, 144, 66

Keel, W. C., Maksym, W. P., Bennert, V. N., et al. 2015, AJ, 149, 155

Lawrence, A., \& Elvis, M. 2010, ApJ, 714, 561

Lintott, C. J., Schawinski, K., Keel, W., et al. 2009, MNRAS, 399, 129

Lintott, C. J., Schawinski, K., Slosar, A., et al. 2008, MNRAS, 389, 1179
Liu, G., Zakamska, N. L., Greene, J. E., Nesvadba, N. P. H., \& Liu, X. 2013, MNRAS, 430, 2327

MacLeod, C. L., Ross, N. P., Lawrence, A., et al. 2016, MNRAS, in press (arXiv:1509.08393)

Maksym, W. P., Fabbiano, G., Elvis, M., et al. 2017, ApJ, in press (arXiv:1611.05880)

Menezes, R. B., Steiner, J. E., \& da Silva, P. 2016, ApJ, 817, 150

Morse, J. A., Cecil, G., Wilson, A. S., \& Tsvetanov, Z. I. 1998, ApJ, 505, 159

Muno, M. P., Baganoff, F. K., Brandt, W. N., Park, S., \& Morris, M. R. 2007, ApJL, 656, L69

Netzer, H. 1990, Active Galactic Nuclei (Heidelberg: ARI), 57

Novak, G. S., Ostriker, J. P., \& Ciotti, L. 2011, ApJ, 737, 26

Nussbaumer, H., \& Schmutz, W. 1984, A\&A, 138, 495

Osterbrock, D. E., \& Ferland, G. J. (ed.) 2006, Astrophysics of Gaseous Nebulae and Active Galactic Nuclei (2nd ed.; Sausalito, CA: Univ. Science Books), 22

Penston, M. V., \& Perez, E. 1984, MNRAS, 211, 33

Prieto, M. A., \& Freudling, W. 1996, MNRAS, 279, 63

Rampadarath, H., Garrett, M. A., Józsa, G. I. G., et al. 2010, A\&A, 517, L8

Röttgering, H. J. A., Tang, Y., Bremer, M. A. R., et al. 1996, MNRAS, 282, 1033

Ruan, J. J., Anderson, S. F., Cales, S. L., et al. 2015, arXiv:1509.03634

Runco, J. N., Cosens, M., Bennert, V. N., et al. 2016, ApJ, 821, 33

Runnoe, J. C., Cales, S., Ruan, J. J., et al. 2016, MNRAS, 455, 1691

Sartori, L. F., Schawinski, K., Koss, M., et al. 2016, MNRAS, 457, 3629

Schawinski, K., Koss, M., Berney, S., \& Sartori, L. F. 2015, MNRAS, 451,2517

Schirmer, M., Diaz, R., Holhjem, K., Levenson, N. A., \& Winge, C. 2013, ApJ, 763,60

Schirmer, M., Malhotra, S., Levenson, N. A., et al. 2016, MNRAS, 463, 1554

Schweizer, F., Seitzer, P., Kelson, D. D., Villanueva, E. V., \& Walth, G. L. 2013, ApJ, 773, 148

Shankar, F., Weinberg, D. H., \& Miralda-Escudé, J. 2009, ApJ, 690, 20

Spitzer, L., Jr., \& Greenstein, J. L. 1951, ApJ, 114, 407

Stockton, A., Fu, H., \& Canalizo, G. 2006, NewAR, 50, 694

Storchi-Bergmann, T., Schmitt, H. R., Calzetti, D., \& Kinney, A. L. 1998, AJ, 115,909

van Dokkum, P. G. 2001, PASP, 113, 1420

Véron-Cetty, M. P., \& Véron, P. 2001, A\&A, 375, 791

Wright, E. L., Eisenhardt, P. R. M., Mainzer, A. K., et al. 2010, AJ, 140, 1868

Zubovas, K., King, A. R., \& Nayakshin, S. 2011, MNRAS, 415, L21 